\title{
No strong $C P$ violation up to the one-loop level in a two-Higgs-doublet model
}

\author{
P. M. Ferreira ${ }^{1,2, a}$, L. Lavoura ${ }^{3, b}$ \\ ${ }^{1}$ Instituto Superior de Engenharia de Lisboa-ISEL, 1959-007 Lisboa, Portugal \\ ${ }^{2}$ Centro de Física Teórica e Computacional, Faculdade de Ciências, Universidade de Lisboa, Av. Prof. Gama Pinto 2, $1649-003$ Lisboa, Portugal \\ ${ }^{3}$ Universidade de Lisboa, Instituto Superior Técnico, CFTP, Av. Rovisco Pais 1, 1049-001 Lisboa, Portugal
}

Received: 29 April 2019 / Accepted: 17 June 2019 / Published online: 1 July 2019

(C) The Author(s) 2019

\begin{abstract}
We put forward a two-Higgs-doublet model, furnished with a $\mathbb{Z}_{3}$ symmetry, wherein $C P$ is conserved in the dimension-four terms of the Lagrangian and is softly broken in the scalar potential. The new particles of our model are one neutral scalar $H$, one neutral pseudoscalar $A$, and two charged scalars $H^{ \pm}$. In our model the only locus of $C P$ violation is the CKM matrix. Strong $C P$ violation is absent both at the tree and one-loop levels. We work out the phenomenological constraints on our model, which features flavour-changing neutral Yukawa interactions, showing that the new scalar particles may in some cases be lighter than $500 \mathrm{GeV}$.
\end{abstract}

\section{Introduction}

Non-perturbative effects in Quantum Chromodynamics (QCD) may lead to $P$ and $C P$ violation, characterized by a parameter $\theta$, in hadronic processes. The experimental upper bound on the electric dipole moment of the neutron necessitates $\theta \lesssim 10^{-9} .1$ The presence in the Lagrangian of this unnaturally small parameter is known as the "strong $C P$ problem'.

The angle $\theta$ is the sum of two terms, $\theta_{\mathrm{QCD}}$ and $\theta_{\mathrm{QFD}}$. Here, $\theta_{\mathrm{QCD}}$ is the value of a $P$ - and $C P$-violating angle in the QCD vacuum, and $\theta_{\mathrm{QFD}}$ originates in the chiral rotation of the quark fields needed to render the quark masses real and positive. Let $p$ and $n$ denote the three up-type quarks and the three down-type quarks, respectively, in a weak basis. Let the mass terms of those quarks be given by

\footnotetext{
${ }^{1}$ For a recente estimate of the maximum possible value of $\theta$, see Ref. [1].

a e-mail: pmmferreira@fc.ul.pt

be-mail: balio@cftp.tecnico.ulisboa.pt
}

$\mathcal{L}_{\text {mass }}=-\bar{p}_{L} M_{p} p_{R}-\bar{n}_{L} M_{n} n_{R}+$ H.c.,

where $M_{p}$ and $M_{n}$ are $3 \times 3$ matrices in flavour space. Then, $\theta_{\mathrm{QFD}}=\arg \operatorname{det}\left(M_{p} M_{n}\right)$.

There are two general approaches to solving the strong $C P$ problem. In the first approach it is claimed that $\theta$ has no significance or physical consequences; theories with different values of $\theta$ are equivalent and one may set $\theta$ to zero without loss of generality. This may happen either because one of the quarks is massless ${ }^{2}$ or because of the presence in the theory of a Peccei-Quinn symmetry [3]; there are also claims that QCD dynamics itself cures the strong $C P$ problem. ${ }^{3}$ The second approach, which we shall follow, acknowledges the strong $C P$ problem and tries to find some symmetry that naturally leads to the smallness of $\theta$. One firstly assumes the dimension-four part of the Lagrangian to be either $C P$ symmetric or $P$-symmetric; this assumption sets $\theta_{\mathrm{QCD}}$ to zero. The $C P$ or $P$ symmetry must be either softly or spontaneously broken; one performs this breaking in such a way that $\theta_{\mathrm{QFD}}$ turns out to be zero at the tree level, because of some peculiar form of $M_{p}$ and $M_{n}$. Still, it is difficult to avoid loop contributions to $\theta_{\mathrm{QFD}}$ arising from the quark self-energies $\Sigma$; they add to the tree-level mass matrices $M$ and then

$\arg \operatorname{det}(M+\Sigma) \approx \operatorname{Im}\left[\operatorname{tr}\left(M^{-1} \Sigma\right)\right]$

is in general nonzero. Artful models are able to obtain $\operatorname{Im}\left[\operatorname{tr}\left(M_{p}^{-1} \Sigma_{p}\right)\right]+\operatorname{Im}\left[\operatorname{tr}\left(M_{n}^{-1} \Sigma_{n}\right)\right]$ equal to zero at the oneloop level and sometimes even at the two-loop level.

There are various ways to achieve quark mass matrices displaying $\arg \operatorname{det}\left(M_{p} M_{n}\right)=0$. Most of those ways, col-

\footnotetext{
${ }^{2}$ For a recent speculation that some quarks may be massless, see Ref. [2].

${ }^{3}$ For a recent instance of such a claim, see Ref. [4].
} 
lectively known as Barr-Nelson-type models ${ }^{4}$, employ extra quarks. There are also many models for solving the strong $C P$ problem that use extra gauge symmetries, especially the left-right symmetry $S U(2)_{\mathrm{L}} \times S U(2)_{\mathrm{R}} \times U(1)_{B-L}$. In this paper we propose a simple extension of the Standard Model $(\mathrm{SM})$, with gauge group $S U(2)_{\mathrm{L}} \times U(1)$ and without any extra fermions, that partially solves the strong $C P$ problem. Our model is a two-Higgs-doublet model (2HDM) [6].

In a $2 \mathrm{HDM}$ the quark Yukawa Lagrangian is

$$
\begin{aligned}
& \mathcal{L}_{\text {Yukawa }}=-\sum_{j, k=1}^{3} \sum_{a=1}^{2} \bar{Q}_{L j} \\
& \times\left[\Phi_{a}\left(\Gamma_{a}\right)_{j k} n_{R k}+\tilde{\Phi}_{a}\left(\Delta_{a}\right)_{j k} p_{R k}\right]+\text { H.c. }
\end{aligned}
$$

where $\Phi_{a}=\left(\phi_{a}^{+}, \phi_{a}^{0}\right)^{T}$ and $\tilde{\Phi}_{a}=\left(\phi_{a}^{0^{*}},-\phi_{a}^{-}\right)^{T}$ for $a=$ 1,2 are scalar doublets of $S U(2)_{\mathrm{L}}$. Furthermore, $\bar{Q}_{L j}=$ $\left(\bar{p}_{L j}, \bar{n}_{L j}\right)$, and the $\Gamma_{a}$ and $\Delta_{a}$ are four $3 \times 3$ matrices in flavour space containing the Yukawa coupling constants. We expand the scalar doublets as

$\Phi_{a}=e^{i \aleph_{a}}\left(\begin{array}{c}\phi_{a}^{+} \\ \left(v_{a}+\rho_{a}+i \eta_{a}\right) / \sqrt{2}\end{array}\right)$,

where $v_{a} \exp \left(i \aleph_{a}\right) / \sqrt{2}=\left\langle 0\left|\phi_{a}^{0}\right| 0\right\rangle$ and the $v_{a}$ are nonnegative real by definition. We define $v=\sqrt{v_{1}^{2}+v_{2}^{2}}=$ $2 m_{W} / g=246 \mathrm{GeV}$ and $\tan \beta \equiv v_{2} / v_{1}$; then,

$\frac{v_{1}}{v}=\cos \beta, \quad \frac{v_{2}}{v}=\sin \beta$,

where the angle $\beta$ is in the first quadrant. There is one physical pseudoscalar $A$ and one unphysical (Goldstone boson) pseudoscalar $G^{0}$ :

$\left(\begin{array}{c}G^{0} \\ A\end{array}\right)=\left(\begin{array}{cc}c_{\beta} & s_{\beta} \\ s_{\beta} & -c_{\beta}\end{array}\right)\left(\begin{array}{l}\eta_{1} \\ \eta_{2}\end{array}\right)$.

(From now on, $s \xi \equiv \sin \xi$ and $c \xi \equiv \cos \xi$ for any needed angle $\xi$ ). There is a pair of physical charged scalars $H^{ \pm}$and a pair of unphysical (Goldstone bosons) charged scalars $G^{ \pm}$:

$\left(\begin{array}{c}G^{ \pm} \\ H^{ \pm}\end{array}\right)=\left(\begin{array}{cc}c_{\beta} & s_{\beta} \\ s_{\beta} & -c_{\beta}\end{array}\right)\left(\begin{array}{c}\phi_{1}^{ \pm} \\ \phi_{2}^{ \pm}\end{array}\right)$

There are two physical neutral scalars $h$ and $H$ :

$\left(\begin{array}{c}h \\ H\end{array}\right)=\left(\begin{array}{c}s_{\alpha}-c_{\alpha} \\ -c_{\alpha}-s_{\alpha}\end{array}\right)\left(\begin{array}{c}\rho_{1} \\ \rho_{2}\end{array}\right)$.

The neutral scalar $h$ is chosen to coincide with the LHCobserved particle with mass $125 \mathrm{GeV}$. In our specific $2 \mathrm{HDM}$

\footnotetext{
${ }^{4}$ For a recent example of one such model, see Ref. [6]
}

there are softly-broken $C P$ and $\mathbb{Z}_{3}$ symmetries such that $h$ and $H$ do not mix with $A$. The interaction Lagrangian between a scalar and a pair of gauge bosons is

$$
\begin{aligned}
\mathcal{L}_{S V V} & =\frac{g}{v}\left(m_{W} W_{\xi}^{-} W^{\xi+}+\frac{m_{Z}}{2 c_{\theta_{w}}} Z_{\xi} Z^{\xi}\right)\left(v_{1} \rho_{1}+v_{2} \rho_{2}\right) \\
& =-g\left(m_{W} W_{\xi}^{-} W^{\xi+}+\frac{m_{Z}}{2 c_{\theta_{w}}} Z_{\xi} Z^{\xi}\right)\left(h s_{\beta-\alpha}+H c_{\beta-\alpha}\right),
\end{aligned}
$$

where $\theta_{w}$ is Weinberg's angle. Because of the LHC data we now know that $\left|s_{\beta-\alpha}\right| \approx 1$.

The quark mass matrices are

$M_{n}=\frac{v_{1} e^{i \aleph_{1}} \Gamma_{1}+v_{2} e^{i \aleph_{2}} \Gamma_{2}}{\sqrt{2}}$,

$M_{p}=\frac{v_{1} e^{-i \aleph_{1}} \Delta_{1}+v_{2} e^{-i \aleph_{2}} \Delta_{2}}{\sqrt{2}}$.

Let the unitary matrices $U_{L, R}^{n, p}$ bi-diagonalize $M_{n}$ and $M_{p}$ as

$U_{L}^{n \dagger} M_{n} U_{R}^{n}=M_{d} \equiv \operatorname{diag}\left(m_{d}, m_{s}, m_{b}\right)$,

$U_{L}^{p^{\dagger}} M_{p} U_{R}^{p}=M_{u} \equiv \operatorname{diag}\left(m_{u}, m_{c}, m_{t}\right)$.

The CKM matrix is

$V=U_{L}^{p^{\dagger}} U_{L}^{n}$

We define

$N_{n}=\frac{v_{2} e^{i \aleph_{1}} \Gamma_{1}-v_{1} e^{i \aleph_{2}} \Gamma_{2}}{\sqrt{2}}$,

$N_{p}=\frac{v_{2} e^{-i \aleph_{1}} \Delta_{1}-v_{1} e^{-i \aleph_{2}} \Delta_{2}}{\sqrt{2}}$,

and

$N_{d}=U_{L}^{n \dagger} N_{n} U_{R}^{n}$,

$N_{u}=U_{L}^{p \dagger} N_{p} U_{R}^{p}$.

Then, the Yukawa interactions in the physical basis are given by

$$
\begin{aligned}
\mathcal{L}_{\text {physical }}= & \frac{i A}{v} \bar{u}\left(N_{u} P_{R}-N_{u}^{\dagger} P_{L}\right) u \\
& +\frac{i A}{v} \bar{d}\left(N_{d}^{\dagger} P_{L}-N_{d} P_{R}\right) d \\
& +\frac{h}{v} \bar{u}\left[\left(s_{\beta-\alpha} M_{u}-c_{\beta-\alpha} N_{u}^{\dagger}\right) P_{L}\right. \\
& \left.+\left(s_{\beta-\alpha} M_{u}-c_{\beta-\alpha} N_{u}\right) P_{R}\right] u \\
+ & \frac{h}{v} \bar{d}\left[\left(s_{\beta-\alpha} M_{d}-c_{\beta-\alpha} N_{d}^{\dagger}\right) P_{L}\right. \\
& \left.+\left(s_{\beta-\alpha} M_{d}-c_{\beta-\alpha} N_{d}\right) P_{R}\right] d \\
& +\frac{H}{v} \bar{u}\left[\left(c_{\beta-\alpha} M_{u}+s_{\beta-\alpha} N_{u}^{\dagger}\right) P_{L}\right. \\
& \left.+\left(c_{\beta-\alpha} M_{u}+s_{\beta-\alpha} N_{u}\right) P_{R}\right] u
\end{aligned}
$$




$$
\begin{aligned}
& +\frac{H}{v} \bar{d}\left[\left(c_{\beta-\alpha} M_{d}+s_{\beta-\alpha} N_{d}^{\dagger}\right) P_{L}\right. \\
& \left.+\left(c_{\beta-\alpha} M_{d}+s_{\beta-\alpha} N_{d}\right) P_{R}\right] d \\
& +\frac{\sqrt{2} H^{+}}{v} \bar{u}\left(N_{u}^{\dagger} V P_{L}-V N_{d} P_{R}\right) d \\
& +\frac{\sqrt{2} H^{-}}{v} \bar{d}\left(V^{\dagger} N_{u} P_{R}-N_{d}^{\dagger} V^{\dagger} P_{L}\right) u,
\end{aligned}
$$

where $P_{L}=\left(1-\gamma_{5}\right) / 2$ and $P_{R}=\left(1+\gamma_{5}\right) / 2$ are the projectors of chirality. Also, $u$ and $d$ are column vectors subsuming the fields of the physical up-type and down-type quarks, respectively. In Eq. (15) we have omitted the Yukawa interactions of the Goldstone bosons; they have the same Lagrangian as in the SM.

In this paper we put forward a $2 \mathrm{HDM}$ where $C P$ is conserved in the dimension-four terms of the Lagrangian, hence $\theta_{\mathrm{QCD}}=0$, and $\operatorname{det}\left(M_{p} M_{n}\right)$ is real both because of $C P$ and because of a $\mathbb{Z}_{3}$ symmetry. (Another symmetry-furnished $2 \mathrm{HDM}$ that also purported to alleviate the strong $C P$ problem was proposed long time ago [7]). Remarkably, our model provides for the absence of strong $C P$ violation even at the one-loop level.

In Sect. 2 we explain our model. In Sect. 3 we demonstrate that strong $C P$ violation vanishes at the one-loop level in our model. Section 4 is devoted to the phenomenological constraints on the model. Section 5 contains the main conclusions of our work. Appendices A, B, and C present some formulas used in the analysis of Sect. 4. Appendix D gives a benchmark point for the parameters of the model.

\section{The model}

Our model is a $2 \mathrm{HDM}$ supplemented by the standard $C P$ symmetry and by a $\mathbb{Z}_{3}$ symmetry. Let $\omega=\exp (2 i \pi / 3)$, then the $\mathbb{Z}_{3}$ symmetry reads

$$
\begin{aligned}
& \Phi_{2} \rightarrow \omega^{2} \Phi_{2}, \\
& Q_{L 1} \rightarrow \omega^{2} Q_{L 1}, \quad Q_{L 2} \rightarrow \omega Q_{L 2}, \\
& n_{R 3} \rightarrow \omega n_{R 3}, \\
& p_{R 1} \rightarrow \omega p_{R 1}, \quad p_{R 2} \rightarrow \omega p_{R 2} .
\end{aligned}
$$

This represents just a slight change from the $\mathbb{Z}_{3}$ symmetry of the 2HDM of Ref. [8].

Both $C P$ and $\mathbb{Z}_{3}$ are softly broken by terms in the quadratic part of the scalar potential. Soft breaking of a symmetry consists in that symmetry holding in all the Lagrangian terms of dimension higher than some value, but not holding for the Lagrangian terms of dimension smaller than, or equal to, that value. In our case, both $C P$ and $\mathbb{Z}_{3}$ hold for terms of dimension four but are broken by terms of dimension two, viz. by the terms with coefficient $\mu_{3}$ in line (17a). In principle, a model with a softly broken symmetry should eventually be justified through an ultraviolet completion, viz. a more complete model, with extra fields active at higher energies, which effectively mimics at low energy scales the model with the softly-broken symmetry. Unfortunately, such a ultraviolet completion is often quite difficult to construct explicitly - we attempted such a construction by adding singlet scalar fields to the theory, who would develop vevs at some high scale and then be "integrated out", leaving the desired low energy potential with only two doublets. However, we were unable to build such extensions that left both the $C P$ and $\mathbb{Z}_{3}$ symmetries intact at low energies - which of course does not mean such an UV completion does not exist. In the absence of any such explicit construction, a softly broken symmetry constitutes a strong, non-trivial assumption. This is, certainly, a weakness of the model in this paper.

The softly broken $C P$ and $\mathbb{Z}_{3}$ scalar potential is written as

$$
\begin{aligned}
V= & \mu_{1} \Phi_{1}^{\dagger} \Phi_{1}+\mu_{2} \Phi_{2}^{\dagger} \Phi_{2} \\
& -\mu_{3}\left(e^{-i \aleph} \Phi_{1}^{\dagger} \Phi_{2}+e^{i \aleph} \Phi_{2}^{\dagger} \Phi_{1}\right) \\
& +\frac{\lambda_{1}}{2}\left(\Phi_{1}^{\dagger} \Phi_{1}\right)^{2}+\frac{\lambda_{2}}{2}\left(\Phi_{2}^{\dagger} \Phi_{2}\right)^{2} \\
& +\lambda_{3} \Phi_{1}^{\dagger} \Phi_{1} \Phi_{2}^{\dagger} \Phi_{2}+\lambda_{4} \Phi_{1}^{\dagger} \Phi_{2} \Phi_{2}^{\dagger} \Phi_{1},
\end{aligned}
$$

where $\mu_{3}$ is real and positive by definition. The terms with coefficient $\mu_{3}$ break the symmetry $\mathbb{Z}_{3}$ softly. The phase $\aleph$ breaks $C P$ softly.

The vacuum expectation values $\left\langle 0\left|\phi_{a}^{0}\right| 0\right\rangle=v_{a} \exp \left(i \aleph_{a}\right) /$ $\sqrt{2}$ have phases $\aleph_{a}$ such that $\aleph_{2}-\aleph_{1}=\aleph$ offsets the phase $-\aleph$ of the term $-\mu_{3} e^{-i \aleph} \Phi_{1}^{\dagger} \Phi_{2}$ of the scalar potential. Thus, there is one gauge-invariant vacuum phase that offsets one phase in the potential, with the consequence that the potential of the physical scalar fields is $C P$-invariant, in particular there is no mixing between the scalars $h$ and $H$ and the pseudoscalar $A$. The stationarity equations for the vacuum are

$$
\begin{aligned}
\mu_{1} v_{1}^{2}+\frac{\lambda_{1}}{2} v_{1}^{4} & =\mu_{2} v_{2}^{2}+\frac{\lambda_{2}}{2} v_{2}^{4} \\
& =\mu_{3} v_{1} v_{2}-\frac{\lambda_{3}+\lambda_{4}}{2} v_{1}^{2} v_{2}^{2} .
\end{aligned}
$$

Referring to Eq. (4) and defining

$T_{a}=\frac{\rho_{a}^{2}+\eta_{a}^{2}}{2}+\phi_{a}^{-} \phi_{a}^{+}$

for $a=1,2$, the potential is then

$$
\begin{aligned}
V= & -\frac{\lambda_{1} v_{1}^{4}+\lambda_{2} v_{2}^{4}}{8}-\frac{\lambda_{3}+\lambda_{4}}{4} v_{1}^{2} v_{2}^{2} \\
& +\mu_{3}\left(\frac{v_{2}}{v_{1}} T_{1}+\frac{v_{1}}{v_{2}} T_{2}-\rho_{1} \rho_{2}\right. \\
& \left.-\eta_{1} \eta_{2}-\phi_{1}^{-} \phi_{2}^{+}-\phi_{2}^{-} \phi_{1}^{+}\right)
\end{aligned}
$$




$$
\begin{aligned}
& +\sum_{a=1}^{2} \frac{\lambda_{a}}{2}\left(v_{a} \rho_{a}+T_{a}\right)^{2} \\
& +\left(\lambda_{3}+\lambda_{4}\right)\left(v_{1} \rho_{1}+T_{1}\right)\left(v_{2} \rho_{2}+T_{2}\right) \\
& -\frac{\lambda_{4}}{2}\left\{\left[\left(v_{1}+\rho_{1}\right) \phi_{2}^{-}-\left(v_{2}+\rho_{2}\right) \phi_{1}^{-}\right]\right. \\
& \times\left[\left(v_{1}+\rho_{1}\right) \phi_{2}^{+}-\left(v_{2}+\rho_{2}\right) \phi_{1}^{+}\right] \\
& +\left(\eta_{1} \phi_{2}^{-}-\eta_{2} \phi_{1}^{-}\right)\left(\eta_{1} \phi_{2}^{+}-\eta_{2} \phi_{1}^{+}\right) \\
& +i\left(\phi_{2}^{-} \phi_{1}^{+}-\phi_{1}^{-} \phi_{2}^{+}\right)\left[\eta_{1}\left(v_{2}+\rho_{2}\right)\right. \\
& \left.\left.-\eta_{2}\left(v_{1}+\rho_{1}\right)\right]\right\} .
\end{aligned}
$$

The potential (20) is invariant under the $C P$ transformation

$$
C P: \quad\left\{\begin{array}{l}
\phi_{a}^{-}(x) \rightarrow \exp (i \lambda) \phi_{a}^{+}(\bar{x}), \\
\phi_{a}^{+}(x) \rightarrow \exp (-i \lambda) \phi_{a}^{-}(\bar{x}), \\
\rho_{a}(x) \rightarrow \rho_{a}(\bar{x}), \\
\eta_{a}(x) \rightarrow-\eta_{a}(\bar{x}),
\end{array}\right.
$$

for $a=1,2$, where $x=(t, \mathbf{r})$ and $\bar{x}=(t,-\mathbf{r})$. The phase $\lambda$ in the $C P$ transformation (21) is arbitrary.

The physical potential contains seven parameters $\mu_{1,2,3}$ and $\lambda_{1,2,3,4}$, since the phase $\aleph$ in line (17a) is cancelled out by the vacuum phase. Instead of those seven parameters we will use as input $v=246 \mathrm{GeV}, m_{h}=125 \mathrm{GeV}$, the angles $\alpha$ and $\beta$, and the masses $m_{H}$ of $H, m_{A}$ of $A$, and $m_{H^{+}}$of $H^{ \pm}$. Then [9],

$$
\begin{aligned}
& \mu_{3}=m_{A}^{2} s_{\beta} c_{\beta}, \\
& \lambda_{1}=\frac{-m_{A}^{2} s_{\beta}^{2}+m_{h}^{2} s_{\alpha}^{2}+m_{H}^{2} c_{\alpha}^{2}}{v^{2} c_{\beta}^{2}}, \\
& \lambda_{2}=\frac{-m_{A}^{2} c_{\beta}^{2}+m_{h}^{2} c_{\alpha}^{2}+m_{H}^{2} s_{\alpha}^{2}}{v^{2} s_{\beta}^{2}}, \\
& \lambda_{3}=\frac{2 m_{H^{+}}^{2}-m_{A}^{2}}{v^{2}}+\frac{\left(m_{H}^{2}-m_{h}^{2}\right) s_{\alpha} c_{\alpha}}{v^{2} s_{\beta} c_{\beta}}, \\
& \lambda_{4}=\frac{2\left(m_{A}^{2}-m_{H^{+}}^{2}\right)}{v^{2}} .
\end{aligned}
$$

In order for the potential to be bounded from below, one must impose the conditions [6]

$\lambda_{1}>0, \quad \lambda_{2}>0, \quad \lambda_{3}>-\sqrt{\lambda_{1} \lambda_{2}}, \quad \lambda_{3}+\lambda_{4}>-\sqrt{\lambda_{1} \lambda_{2}}$.

In order to avoid the situation of 'panic vacuum' [9] one must enforce the condition [10]

$\frac{2 \mu_{3}}{v_{1} v_{2}}>\lambda_{3}+\lambda_{4}-\sqrt{\lambda_{1} \lambda_{2}}$.

The conditions in order for tree-level unitarity not to be violated are

$$
\left|\lambda_{1}\right|<8 \pi
$$

$$
\begin{aligned}
& \left|\lambda_{2}\right|<8 \pi \\
& \left|\lambda_{3}\right|<8 \pi \\
& \left|\lambda_{3}+\lambda_{4}\right|<8 \pi \\
& \left|\lambda_{3}-\lambda_{4}\right|<8 \pi \\
& \left|\lambda_{3}+2 \lambda_{4}\right|<8 \pi \\
& \left|\lambda_{1}+\lambda_{2}+\sqrt{\left(\lambda_{1}-\lambda_{2}\right)^{2}+4 \lambda_{4}^{2}}\right|<16 \pi \\
& \left|3 \lambda_{1}+3 \lambda_{2}+\sqrt{9\left(\lambda_{1}-\lambda_{2}\right)^{2}+4\left(2 \lambda_{3}+\lambda_{4}\right)^{2}}\right|<16 \pi
\end{aligned}
$$

Because of the $\mathbb{Z}_{3}$ symmetry (16), the matrices $\Gamma_{a}$ and $\Delta_{a}$ are

$\Gamma_{1}=\left(\begin{array}{ccc}0 & 0 & 0 \\ 0 & 0 & b_{1} \\ d_{1} & f_{1} & 0\end{array}\right), \quad \Gamma_{2}=\left(\begin{array}{ccc}d_{2} & f_{2} & 0 \\ 0 & 0 & 0 \\ 0 & 0 & b_{2}\end{array}\right)$

$\Delta_{1}=\left(\begin{array}{ccc}0 & 0 & 0 \\ p_{1} & q_{1} & 0 \\ 0 & 0 & r_{1}\end{array}\right), \Delta_{2}=\left(\begin{array}{ccc}p_{2} & q_{2} & 0 \\ 0 & 0 & r_{2} \\ 0 & 0 & 0\end{array}\right)$.

The dimensionless numbers $b_{a}, d_{a}, f_{a}, p_{a}, q_{a}$, and $r_{a}(a=$ $1,2)$ are real because of the $C P$ symmetry. Clearly,

$M_{n}=\frac{1}{\sqrt{2}}\left(\begin{array}{ccc}d_{2} v_{2} e^{i \aleph_{2}} & f_{2} v_{2} e^{i \aleph_{2}} & 0 \\ 0 & 0 & b_{1} v_{1} e^{i \aleph_{1}} \\ d_{1} v_{1} e^{i \aleph_{1}} & f_{1} v_{1} e^{i \aleph_{1}} & b_{2} v_{2} e^{i \aleph_{2}}\end{array}\right)$

$M_{p}=\frac{1}{\sqrt{2}}\left(\begin{array}{ccc}p_{2} v_{2} e^{-i \aleph_{2}} & q_{2} v_{2} e^{-i \aleph_{2}} & 0 \\ p_{1} v_{1} e^{-i \aleph_{1}} & q_{1} v_{1} e^{-i \aleph_{1}} & r_{2} v_{2} e^{-i \aleph_{2}} \\ 0 & 0 & r_{1} v_{1} e^{-i \aleph_{1}}\end{array}\right)$.

Therefore

$\operatorname{det}\left(M_{n} M_{p}\right)=\frac{v_{1}^{4} v_{2}^{2}}{8} b_{1}\left(d_{1} f_{2}-d_{2} f_{1}\right) r_{1}\left(p_{2} q_{1}-p_{1} q_{2}\right)$

is real, hence $\theta_{\mathrm{QFD}}=0$. Because of the assumed $C P$ invariance of the quartic part of the Lagrangian, $\theta_{\mathrm{QCD}}=0$ too. Thus, $\theta=\theta_{\mathrm{QCD}}+\theta_{\mathrm{QFD}}=0$, i.e. there is no strong $C P$ violation at the tree level.

We now define

$$
\begin{aligned}
& \beta_{a} \equiv \arg b_{a}, \quad \delta_{a} \equiv \arg d_{a}, \quad \varphi_{a} \equiv \arg f_{a}, \\
& \pi_{a} \equiv \arg p_{a}, \quad \chi_{a} \equiv \arg q_{a}, \quad \varrho_{a} \equiv \arg r_{a},
\end{aligned}
$$

for $a=1,2$; and

$$
\begin{aligned}
& \Delta \beta \equiv \beta_{2}-\beta_{1}, \quad \Delta \delta \equiv \delta_{2}-\delta_{1}, \quad \Delta \varphi \equiv \varphi_{2}-\varphi_{1} \\
& \Delta \pi \equiv \pi_{2}-\pi_{1}, \quad \Delta \chi \equiv \chi_{2}-\chi_{1}, \quad \Delta \varrho \equiv \varrho_{2}-\varrho_{1} .
\end{aligned}
$$

All the phases in equations (29) and (30) are either 0 or $\pi$ because $b_{a}, d_{a}, \ldots, r_{a}$ are real. We define the diagonal matrices 


$$
\begin{aligned}
& X_{L n}=\operatorname{diag}\left(1, e^{i(2 \aleph+\Delta \delta+\Delta \beta)}, e^{i(\aleph+\Delta \delta)}\right), \\
& X_{R n}=\operatorname{diag}\left(e^{i\left(-\aleph_{2}-\delta_{2}\right)}, e^{i\left(-\aleph_{2}-\varphi_{2}\right)}, e^{i\left(\aleph_{1}-2 \aleph_{2}-\Delta \delta-\beta_{2}\right)}\right), \\
& X_{L p}=\operatorname{diag}\left(1, e^{i(-\aleph+\Delta \pi)}, e^{i(-2 \aleph+\Delta \pi+\Delta \varrho)}\right), \\
& X_{R p}=\operatorname{diag}\left(e^{i\left(\aleph_{2}-\pi_{2}\right)}, e^{i\left(\aleph_{2}-\chi_{2}\right)}, e^{i\left(2 \aleph_{2}-\aleph_{1}-\Delta \pi-\varrho_{2}\right)}\right)
\end{aligned}
$$

where $\aleph=\aleph_{2}-\aleph_{1}$. We then have

$$
\begin{aligned}
X_{L n} M_{n} X_{R n} & =\frac{1}{\sqrt{2}}\left(\begin{array}{ccc}
\left|d_{2} v_{2}\right| & \left|f_{2} v_{2}\right| & 0 \\
0 & 0 & \left|b_{1} v_{1}\right| \\
\left|d_{1} v_{1}\right| & \left|f_{1} v_{1}\right| e^{i(\Delta \delta-\Delta \varphi)} & \left|b_{2} v_{2}\right|
\end{array}\right) \\
& \equiv M_{n}^{\prime}, \\
X_{L p} M_{p} X_{R p} & =\frac{1}{\sqrt{2}}\left(\begin{array}{ccc}
\left|p_{2} v_{2}\right| & \left|q_{2} v_{2}\right| & 0 \\
\left|p_{1} v_{1}\right| & \left|q_{1} v_{1}\right| e^{i(\Delta \pi-\Delta \chi)} & \left|r_{2} v_{2}\right| \\
0 & 0 & \left|r_{1} v_{1}\right|
\end{array}\right) \\
& \equiv M_{p}^{\prime} .
\end{aligned}
$$

The matrices $M_{n}^{\prime}$ and $M_{p}^{\prime}$ are real, therefore they may be bi-diagonalized through real orthogonal matrices $O_{L n}, O_{R n}$, $O_{L p}$, and $O_{R p}$ as

$$
O_{L n} M_{n}^{\prime} O_{R n}=M_{d}, \quad O_{L p} M_{p}^{\prime} O_{R p}=M_{u}
$$

Therefore, in the notation of Eq. (11),

$$
\begin{aligned}
& U_{L}^{n \dagger}=O_{L n} X_{L n}, \quad U_{R}^{n}=X_{R n} O_{R n}, \\
& U_{L}^{p^{\dagger}}=O_{L p} X_{L p} \quad U_{R}^{p}=X_{R p} O_{R p} .
\end{aligned}
$$

The CKM matrix is then

$$
\begin{aligned}
V & =O_{L p} \times \operatorname{diag} \\
& \times\left(1, e^{i(-3 \aleph+\Delta \pi-\Delta \delta-\Delta \beta)}, e^{i(-3 \aleph+\Delta \pi-\Delta \delta+\Delta \varrho)}\right) \times O_{L n}^{T} .
\end{aligned}
$$

One sees that the CKM matrix is complex because of the presence of the phase 3 א.

When we compute the matrices $N_{p}$ and $N_{n}$ defined in Eq. (13), we find that

$$
\begin{aligned}
X_{L n} N_{n} X_{R n} & =\frac{1}{\sqrt{2}}\left(\begin{array}{ccc}
-\left|d_{2} v_{1}\right| & -\left|f_{2} v_{1}\right| & 0 \\
0 & 0 & \left|b_{1} v_{2}\right| \\
\left|d_{1} v_{2}\right| & \left|f_{1} v_{2}\right| e^{i(\Delta \delta-\Delta \varphi)} & -\left|b_{2} v_{1}\right|
\end{array}\right) \\
& \equiv N_{n}^{\prime},
\end{aligned}
$$

$$
\begin{aligned}
X_{L p} N_{p} X_{R p} & =\frac{1}{\sqrt{2}}\left(\begin{array}{ccc}
-\left|p_{2} v_{1}\right| & -\left|q_{2} v_{1}\right| & 0 \\
\left|p_{1} v_{2}\right| & \left|q_{1} v_{2}\right| e^{i(\Delta \pi-\Delta \chi)} & -\left|r_{2} v_{1}\right| \\
0 & 0 & \left|r_{1} v_{2}\right|
\end{array}\right) \\
& \equiv N_{p}^{\prime},
\end{aligned}
$$

and then, from Eq. (14),

$N_{d}=O_{L n} N_{n}^{\prime} O_{R n}, \quad N_{u}=O_{L p} N_{p}^{\prime} O_{R p}$

The matrices $N_{d}$ and $N_{u}$ are real.

Thus, in our model

1. The CKM matrix is complex.

2. The matrices $N_{u}$ and $N_{d}$ are real.

3. There is one pseudoscalar $A$ that does not mix with the scalars $h$ and $H$.

4. There is no $C P$ violation in the cubic and quartic interactions of the scalars.

In our model $C P$ violation is located solely in the CKM matrix and originates entirely in the phase $3 \aleph$. This is the same that happened in the model of Ref. [8]; however, in that model there was strong $C P$ violation, while in the present model strong $C P$ violation is absent at the tree level.

\section{No strong $C P$ violation at the one-loop level}

At one-loop level the diagonal and real quark mass matrices $M_{q}$ (where $q$ may be either $u$ or $d$ ) get corrected by self energy diagrams: $M_{q} \rightarrow M_{q}+\Sigma_{q}$. If the diagonal elements of $\Sigma_{q}$ are complex, then $\operatorname{Im}\left[\operatorname{tr}\left(M_{q}^{-1} \Sigma_{q}\right)\right]$ may be nonzero and strong $C P$ violation may arise.

In our model there are no complex phases except in the CKM matrix. Since the matrices $N_{q}$ are real, and since the scalars $h$ and $H$ do not mix with the pseudoscalar $A$, the $\Sigma_{q}$ generated through the emission and reabsorption (E\&R) by the quarks of either $h$ or $H$ or $A$ are real, hence innocuous. The same happens with the $\Sigma_{q}$ generated through the E\&R of $Z^{0}$ gauge bosons. On the other hand, diagrams with the E\&R of $W^{ \pm}$gauge bosons do not generate mass renormalization (they just produce wavefunction renormalization), since the coupling of $W^{ \pm}$to the quarks is purely left-handed. Therefore, the only diagrams where the complex matrix $V$ arises, and might produce complex $\Sigma_{q}$, are the ones with E\&R of charged scalars $H^{ \pm}$.

The Yukawa interactions of the charged scalars are given by lines $(15 \mathrm{~g})$ and $(15 \mathrm{~h})$. They contain two complex matrices, $X \equiv N_{u}^{\dagger} V$ and $Y \equiv V N_{d}$. The one-loop self-energy of an up-type quark $u_{\alpha}$ caused by the E\&R of $H^{+}$and a down-type quark $d_{j}$ is 


$$
\begin{aligned}
-i \Sigma_{\alpha}(p)= & \frac{2}{v^{2}} \mu^{4-d} \int \frac{\mathrm{d}^{d} k}{(2 \pi)^{d}} \frac{1}{k^{2}-m_{j}^{2}} \frac{1}{(k-p)^{2}} \\
& \times\left(X_{\alpha j} P_{L}-Y_{\alpha j} P_{R}\right)\left(\not k+m_{j}\right) \\
& \times\left(X_{j \alpha}^{\dagger} P_{R}-Y_{j \alpha}^{\dagger} P_{L}\right) \\
= & \frac{2}{v^{2}} \mu^{4-d} \int \frac{\mathrm{d}^{d} k}{(2 \pi)^{d}} \int_{0}^{1} \mathrm{~d} x \frac{1}{\left(k^{2}-\Delta_{j}\right)^{2}} \\
& \times\left(x\left|X_{\alpha j}\right|^{2} \not p P_{R}+x\left|Y_{\alpha j}\right|^{2} \not p P_{L}\right. \\
& \left.-X_{\alpha j} m_{j} Y_{j \alpha}^{\dagger} P_{L}-Y_{\alpha j} m_{j} X_{j \alpha}^{\dagger} P_{R}\right),
\end{aligned}
$$

where

$$
\Delta_{j}=p^{2} x^{2}+\left(-p^{2}+m_{H^{+}}^{2}-m_{j}^{2}\right) x+m_{j}^{2}
$$

and we perform the computation in a space-time of dimension $d$. Thus, the only potentially complex part of the selfenergy is

$$
\begin{aligned}
& \Sigma_{\alpha}(\not p \rightarrow 0)=-\int_{0}^{1} \mathrm{~d} x \frac{X_{\alpha j} m_{j} Y_{j \alpha}^{\dagger} P_{L}+Y_{\alpha j} m_{j} X_{j \alpha}^{\dagger} P_{R}}{8 \pi^{2} v^{2}} \\
& \quad \times\left(\frac{2}{4-d}-\gamma-\ln \frac{\Delta_{j}}{4 \pi \mu^{2}}\right),
\end{aligned}
$$

where $\gamma$ is Euler-Mascheroni's constant. One must sum the expression in the right-hand side of Eq. (40) over the flavour $j$ of the quark $d_{j}$.

The one-loop value of the strong- $C P$ parameter $\theta$ is $\operatorname{Im}\left[\operatorname{tr}\left(M_{u}^{-1} \Sigma_{u}+M_{d}^{-1} \Sigma_{d}\right)\right]$. The diagonal matrix elements of $M_{u}^{-1} \Sigma_{u}$ are $m_{\alpha}^{-1} \Sigma_{\alpha}(\not p \rightarrow 0)$. Now,

$$
\begin{aligned}
\sum_{\alpha} \frac{X_{\alpha i} Y_{j \alpha}^{\dagger}}{m_{\alpha}} & =\sum_{\alpha}\left(N_{d}^{\dagger} V^{\dagger}\right)_{j \alpha} \frac{1}{m_{\alpha}}\left(N_{u}^{\dagger} V\right)_{\alpha i} \\
& =\left(N_{d}^{\dagger} V^{\dagger} M_{u}^{-1^{\dagger}} N_{u}^{\dagger} V\right)_{j i} \\
& =\left(U_{R}^{n \dagger} N_{n}^{\dagger} M_{p}^{-1 \dagger} N_{p}^{\dagger} U_{L}^{n}\right)_{j i} .
\end{aligned}
$$

Therefore,

$$
\begin{aligned}
& \sum_{\alpha, j} \frac{X_{\alpha j} m_{j} Y_{j \alpha}^{\dagger} f\left(m_{j}^{2}\right)}{m_{\alpha}} \\
& \quad=\sum_{j} f\left(m_{j}^{2}\right) m_{j}\left(U_{R}^{n \dagger} N_{n}^{\dagger} M_{p}^{-1^{\dagger}} N_{p}^{\dagger} U_{L}^{n}\right)_{j j} \\
& \quad=\operatorname{tr}\left[U_{L}^{n} f\left(M_{d} M_{d}^{\dagger}\right) M_{d} U_{R}^{n \dagger} N_{n}^{\dagger} M_{p}^{-1 \dagger} N_{p}^{\dagger}\right] \\
& \quad=\operatorname{tr}\left[f\left(M_{n} M_{n}^{\dagger}\right) M_{n} N_{n}^{\dagger} M_{p}^{-1 \dagger} N_{p}^{\dagger}\right] .
\end{aligned}
$$

One easily finds that, in our model, both matrices $M_{n} M_{n}^{\dagger}$ and $M_{n} N_{n}^{\dagger} M_{p}^{-1^{\dagger}} N_{p}^{\dagger}$, and all of their products too, have a structure of phases of the form $\left(\begin{array}{ccc}0 & 2 \aleph & \aleph \\ -2 \aleph & 0 & -\aleph \\ -\aleph & \aleph & 0\end{array}\right)$,

where $\aleph=\aleph_{2}-\aleph_{1}$. Therefore, the diagonal matrix elements of $f\left(M_{n} M_{n}^{\dagger}\right) M_{n} N_{n}^{\dagger} M_{p}^{-1^{\dagger}} N_{p}^{\dagger}$, and hence its trace, are real, no matter what the function $f$ is.

In this way we have demonstrated that $\operatorname{tr}\left(M_{u}^{-1} \Sigma_{u}\right)$ is real. In a similar way one may show that $\operatorname{tr}\left(M_{d}^{-1} \Sigma_{d}\right)$ is also real, hence strong $C P$ violation vanishes at the one-loop level in our model.

\section{Phenomenological analysis of the model}

\subsection{Constraints}

We proceed to analyse how our model conforms to the experimental results. The model has tree-level flavour-changing neutral currents (FCNC) coupling to the scalars, so there is a wealth of flavour-physics observables that need to be taken into account whilst performing a fit of the model to the experimental data. Our procedure involves a global fit of the model's parameters, simultaneously requiring compliance with the theoretical and experimental bounds from the gauge, scalar, and fermionic sectors.

One may rotate the right-handed quarks $n_{R 1}$ and $n_{R 2}$ between themselves in such a way that the entry $f_{2}$ of the Yukawa-coupling matrix $\Gamma_{2}$ becomes zero. Similarly, one may rotate $p_{R 1}$ and $p_{R 2}$ so that $q_{2}$ becomes zero. ${ }^{5,6}$ We use as input the ten entries $b_{1}, d_{1}, f_{1}, b_{2}, d_{2}, p_{1}, q_{1}, r_{1}, p_{2}$, and $r_{2}$ ( $f_{2}$ and $q_{2}$ are set to zero) of the Yukawa-coupling matrices (26), allowing those entries to be either positive or negative. We further input the $C P$-violating phase $\aleph$. We fit these eleven parameters in order to reproduce the quark masses $[11]^{7}$

$m_{u}=(2.2 \pm 2 \times 0.6) \mathrm{MeV}$,

$m_{d}=(4.7 \pm 2 \times 0.5) \mathrm{MeV}$,

$m_{s}=(96 \pm 2 \times 8) \mathrm{MeV}$,

$m_{c}=(1.28 \pm 0.03) \mathrm{GeV}$,

$m_{b}=(4.18 \pm 0.04) \mathrm{GeV}$,

\footnotetext{
Notice that $n_{R 1}$ and $n_{R 2}$ transform in the same way under the $\mathbb{Z}_{3}$ symmetry (16), and $p_{R 1}$ and $p_{R 2}$ also transform in the same way under that symmetry.

${ }^{6}$ With $f_{2}=q_{2}=0$, the phases $\Delta \varphi$ and $\Delta \chi$ in Eq. (30) become meaningless. That has no impact on our reasonings, in particular the matrix $V$ in Eq. (35) does not depend on those phases.

7 We have doubled the uncertainty intervals quoted in Ref. [11] for the masses of the light quarks $u, d$, and $s$; we have done this because of the large theoretical indefinition, due to QCD considerations, as to what exactly should be interpreted as the value of those masses.
} 
$m_{t}=(173.2 \pm 0.6) \mathrm{GeV}$,

and the CKM-matrix observables [11]

$$
\begin{aligned}
& \left|V_{u s}\right|=0.2243 \pm 0.0005 \\
& \left|V_{c b}\right|=0.0422 \pm 0.0008 \\
& \left|V_{u b}\right|=0.00394 \pm 0.00036 \\
& \gamma \equiv \arg \left(-\frac{V_{u d} V_{u b}^{*}}{V_{c d} V_{c b}^{*}}\right)=(73.5 \pm 5.5)^{\circ} .
\end{aligned}
$$

We furthermore input $\tan \beta=v_{2} / v_{1}$. We compute the matrices $N_{n}^{\prime}$ and $N_{p}^{\prime}$ through Eq. 36), and the matrices $N_{d}$ and $N_{u}$ through Eq. (37). Finally, we input $\alpha$ and get to know $\mathcal{L}_{\text {physical }}$ in Eq. 15). ${ }^{8}$

In the scalar potential (17) there are seven independent parameters $\mu_{1,2,3}$ and $\lambda_{1,2,3,4}$. We input instead the seven observables

1. $v=\sqrt{v_{1}^{2}+v_{2}^{2}}=246 \mathrm{GeV}$, which produces the correct masses for the electroweak gauge bosons $W^{ \pm}$and $Z^{0}$;

2 . the lightest $C P$-even-scalar mass $m_{h}=125 \mathrm{GeV}$, corresponding to the Higgs boson observed at the LHC;

3. the angle $\beta=\arctan \left(v_{2} / v_{1}\right)$;

4. the angle $\alpha$;

5. the remaining scalar masses $-m_{H}$ of the second CP-even scalar, $m_{A}$ of the pseudoscalar, and $m_{H^{+}}$of the charged scalar.

The last five parameters must be found through the fitting procedure. We have constrained $m_{H^{+}}$to be larger than $100 \mathrm{GeV}$ and $m_{H}$ and $m_{A}$ to be larger than $130 \mathrm{GeV}$. We have furthermore assumed all three masses to be smaller than $1.2 \mathrm{TeV}$; values of the masses larger than $1.2 \mathrm{TeV}$ would certainly be allowed by the fitting procedure.

The quartic couplings of the model are determined via Eq. (22). We check that the scalar potential is bounded from below, that it does not have a panic vacuum, and that it satisfies unitarity, viz. we check conditions $(23,24,25)$. The constraints from the electroweak oblique parameters $S$ and $T$ are also imposed, by using the expressions for the 2HDM in refs. $[12,13]$.

We will now go into detail about the further constraints that we have imposed.

- We implement the $b \rightarrow s \gamma$ bound described in Appendix A, including the contributions from both the neutral and the charged scalars.

- The most relevant bounds on the off-diagonal entries of the matrices $N_{d}$ and $N_{u}$ come from flavour-physics

\footnotetext{
8 The angle $\alpha$ may be restricted to lie either in the first quadrant or in the fourth quadrant [6].
}

observables, specifically the $K, B_{d}, B_{s}$, and $D$ neutralmeson mass differences, and the $C P$-violating parameter $\epsilon_{K}$. We detail the computation of those quantities, and the requirements on them that we use in our fit, in Appendix B.

- The $Z \rightarrow b \bar{b}$ constraints described in Appendix C are also taken into account. In this case we use only the charged-scalar contributions; the neutral-scalar ones should be negligible.

- For the regions of parameter space where $m_{t}>m_{q}+$ $m_{H^{+}}, q$ being a down-type quark, or where $m_{t}>m_{q}+$ $m_{S}, q$ being either $c$ or $u$ and $S$ being either $h$ or $H$ or $A$, we require that the branching ratio for each of the kinematically viable $t \rightarrow$ light quark + scalar decays be smaller than $5 \times 10^{-3}$, in accordance with the current results on FCNC top decays and on the total top-quark width [11].

- In order that the scalar $h$ of our model complies with the observational data from the LHC - it should be SM-like in its behaviour-we require that its couplings to the electroweak gauge bosons and to the top and bottom quarks do not deviate significantly from the SM expectations. We achieve this by focusing on the coupling modifiers $\kappa_{X}$ defined as $g_{h Z Z}=\kappa_{Z} g_{h Z Z}^{\mathrm{SM}}, g_{h W W}=\kappa_{W} g_{h W W}^{\mathrm{SM}}$, $g_{h t \bar{t}}=\kappa_{t} g_{h t \bar{t}}^{\mathrm{SM}}$, and $g_{h b \bar{b}}=\kappa_{b} g_{h b \bar{b}}^{\mathrm{SM}}$. In our model $\kappa_{Z}=\kappa_{W} \equiv \kappa_{V}$ and

$$
\begin{aligned}
& \kappa_{V}=s_{\beta-\alpha}, \quad \kappa_{t}=s_{\beta-\alpha}-\frac{c_{\beta-\alpha}\left(N_{u}\right)_{33}}{m_{t}}, \\
& \kappa_{b}=s_{\beta-\alpha}-\frac{c_{\beta-\alpha}\left(N_{d}\right)_{33}}{m_{b}},
\end{aligned}
$$

cf. Eqs. (15c) and (15d). In the first stage of the fit we constrain these couplings to obey $0.8 \leq \kappa_{V} \leq 1,0.8 \leq$ $\kappa_{t} \leq 1.2$, and $0.8 \leq\left|\kappa_{b}\right| \leq 1.2,{ }^{9,10}$ in order to roughly reproduce the LHC results. A second stage of the analysis further constrains these couplings, as detailed below.

A numerical scan of the parameter space of the model, in both the scalar and Yukawa sectors, was performed to discover points that obey all the constraints described above. It must be stressed that we have introduced nowhere in our scan a 'no-fine-tuning' assumption: we have tolerated any set of input values that led to the right outputs, even if either the input values or any intermediate computations displayed either 'fine-tunings' or 'unnatural cancelations'. Strong finetunings are often required in order to fit the $D$-meson mass

\footnotetext{
9 Notice that we allow the possibility of a 'wrong-sign regime' $[14,15]$ in the bottom-quark coupling. However, the combination of cuts applied to the model ends up not allowing for that regime.

${ }^{10}$ Notice that $\kappa_{V}$ and $\kappa_{t}$ must have the same sign, otherwise one would expect a huge variation in the $h$ diphoton width, in disagreement with the SM-like observed values.
} 
difference constraint whenever $m_{H}$ and $m_{A}$ are not very high; for the other constraints, fine-tunings are at most moderate and do not occur at all for many points in our fit. As a matter of fact, even for the $D$-meson mass difference, there are many choices of parameters for which no fine-tuning is necessary and one of the scalars has relatively low mass; one such case is presented in Appendix D.

With those points we have proceeded to compute the LHC production cross sections of the neutral scalars in the model, using the software SusHI $[16,17]$ to include the NNLO QCD corrections. We have limited ourselves to the gluongluon production process, which is the dominant one in the LHC environment. Regarding the vector boson-fusion process, no differences will occur in this model vis a vis the usual 2HDM, as the couplings of the scalars to the gauge bosons are the same in both models.

The results for $h$ are expressed in terms of the ratios

$$
\mu_{X}=\frac{\sigma(p p \rightarrow h) \mathrm{BR}(h \rightarrow X)}{\sigma^{\mathrm{SM}}(p p \rightarrow h) \mathrm{BR}^{\mathrm{SM}}(h \rightarrow X)},
$$

where $X$ may be either $Z^{0} Z^{0}, W^{+} W^{-}, b \bar{b}, \tau \bar{\tau}$, or $\gamma \gamma$. The value $\mu_{X}=1$ indicates exact SM-like behaviour. We require that all the $\mu_{X}$ be within $20 \%$ of 1 , which is a fair description of the current LHC results, taking into account the uncertainties. With this imposition, the ranges of variation of $\kappa_{V}=s_{\beta-\alpha}, \kappa_{t}$, and $\kappa_{b}$ become much smaller than initially allowed in the fit: we obtain $0.929 \leq \kappa_{V} \leq 1$ and $0.952 \leq\left\{\kappa_{t}, \kappa_{b}\right\} \leq 1.04$. For comparison, we will also present results for the tighter constraint $\left|\mu_{X}-1\right|<0.1$.

In principle, we should also consider the leptons. The $\mathbb{Z}_{3}$ symmetry in the quark sector must be extended to the leptonic sector. Since flavour violation with leptons is much more constrained than with quarks, the best choice would be to extend $\mathbb{Z}_{3}$ to the leptonic sector in a way identical to the flavour-preserving $2 \mathrm{HDMs}$, allowing only one of the two doublets $\Phi_{a}$ to couple to the leptons and give them mass. We would then have have two possibilities for the couplings of the scalars to the charged leptons-either $\Phi_{1}$ couples to the charged leptons or $\Phi_{2}$ does. The coupling modifier $\kappa_{\tau}=$ $g_{h \tau \bar{\tau}} / g_{h \tau \bar{\tau}}^{\mathrm{SM}}$ is given by

$\kappa_{\tau}=\frac{\cos \alpha}{\sin \beta}$ and $\kappa_{\tau}=-\frac{\sin \alpha}{\cos \beta}$

for the first and second choices, respectively. For definiteness, in our fit we have adopted the second option in Eq. 48), viz. we have imposed

$0.8<\left|\frac{\sin \alpha}{\cos \beta}\right|<1.2$

to the points in our fit. However, since the extension of our model to the leptonic sector is largely arbitrary, we have refrained from taking into account any other constraints on our model that might arise from processes involving leptons. We point out, though, that flavour-changing constraints from processes like $K_{L} \rightarrow \mu^{+} \mu^{-}$or $B_{s} \rightarrow \mu^{+} \mu^{-}$may pose serious challenges to our model.

\subsection{General results}

The bounds on the scalar sector-unitarity, oblique parameters, and vacuum stability-produce the same contraints on the model's parameters than those found in the usual version of the 2HDM. On the other hand, since the symmetry that we are considering affects in a non-trivial way the quark Yukawa matrices, there are major differences relative to other $2 \mathrm{HDMs}$ when the flavour-physics bounds are imposed. The flavour constraints from meson observables and from the top-quark FCNC decays and total width, previously described, constrain severely the magnitudes of the off-diagonal elements of the matrices $N_{u}$ and $N_{d}$. Our fit achieves to keep FCNC under control even with extra scalars of "low" masses- $H$ and $A$ may have masses below $500 \mathrm{GeV}$. This is in contrast with the often-made assumption that models with tree-level FCNC imply masses above $1 \mathrm{TeV}$; this had already been shown not to necessarily apply in the previous version of the current model [9]. In Ref. [18] it has been argued that contributions from the scalar and pseudoscalar particles ( $H$ and $A$, respectively) to FCNC meson observables tend to cancel each other; we have explicitly observed that, for many points in our fit ( $c f$. the point given in Appendix D), the arguments of Ref. [18] apply, and this is the reason why masses of the extra scalars lower than $1 \mathrm{TeV}$ are possible.

One consequence of the present model is the fact that the scalar $h$, which we have taken to be the $125 \mathrm{GeV}$ state observed at the LHC, has tree-level FCNCs, as indicated by its Yukawa interactions in lines (15c) and (15d). Thus, unlike in the (tree-level) SM, $h$ has the possibility of FCNC decays to final states $s \bar{b}, d \bar{b}, d \bar{s}, u \bar{c}$, and their charge-conjugate states. However, for all the points resulting from our fit, these decays are extremely suppressed-the sum of the branching ratios for all of them being at most $2 \times 10^{-6}$ but usually much lower. Therefore, in our model the FCNC decays of the $125 \mathrm{GeV}$-particle are impossible to observe at the $\mathrm{LHC}$, and almost certainly even at future $e^{+} e^{-}$colliders such as the ILC; the existence of those decays has no measurable impact on the phenomenology of the scalar $h$. The FCNC also raise the possibility of alternative production mechanisms for $h$, such as $d \bar{s} \rightarrow h$ or $u \bar{c} \rightarrow h$; such production channels would be favoured by larger proton PDFs relatively to the SM production mechanism $b \bar{b} \rightarrow h$. However, once again these FCNC processes are found to be extremely small in our fit. According to (15c) and (15d), all the FCNC $h$ interactions are suppressed by their proportionality to $c_{\beta-\alpha}$, which is required to be quite small by the SM-like behaviour 


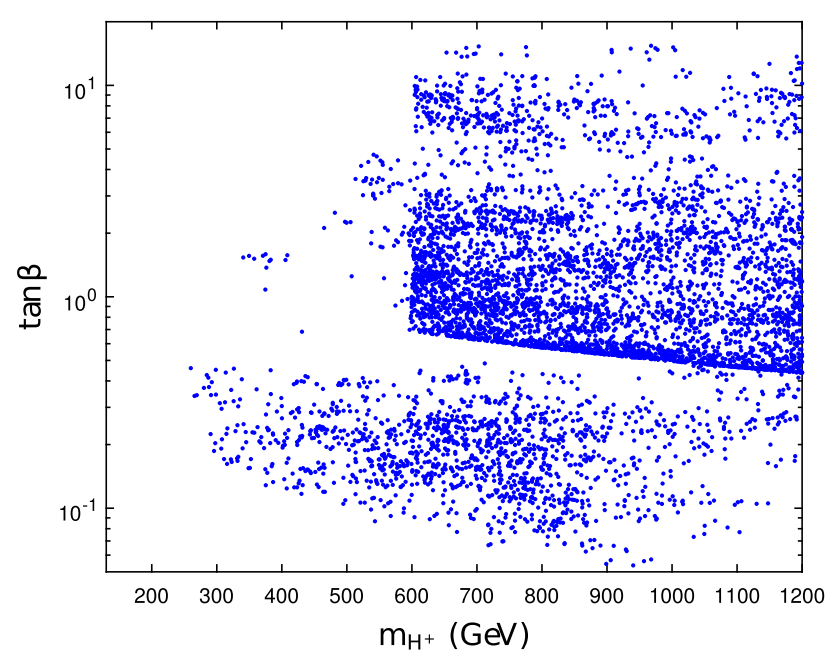

Fig. $1 v_{2} / v_{1}$ versus the charged-Higgs mass for the parameter-space points that survived all the theoretical and experimental constraints

of the $125 \mathrm{GeV}$ scalar $h$, by the ratio between a light-quark mass and $v=246 \mathrm{GeV}$, and, sometimes, by the smallish offdiagonal $N_{d}$ and $N_{u}$ matrix elements induced by compliance with meson-physics bounds.

In Fig. 1 we show the points generated by our fit, displayed on the $\tan \beta-m_{H^{+}}$plane.

(The observed low density of points is merely a consequence of the difficulty in achieving good fits-further searches would yield more points and fill many more regions in the plot; lack of points in some areas has no physical meaning, it is just an artifact of the limited parameter space scan). A clear conclusion from Fig. 1 is that $1 / 20<\tan \beta<20$ in our mode ${ }^{11}$; this is mainly a consequence of the $b \rightarrow s \gamma$ bounds and, to a lesser degree, of the $Z \rightarrow b \bar{b}$ bounds. One also sees in Fig. 1 that values of the charged-scalar mass as low as $130 \mathrm{GeV}$ are easily attained; this is in stark contrast with the findings for the type II $2 \mathrm{HDM}$, where a lower bound on $m_{H^{+}}$of roughly $580 \mathrm{GeV}$ exists. Unlike in the usual typeI and type-II 2HDMs, in our model the quark mass matrices do not emerge from the Yukawa couplings to a single scalar doublet, but rather from the couplings to both $\Phi_{1}$ and $\Phi_{2}$. As such, although we employ the standard definition $\tan \beta=v_{2} / v_{1}$, the usual wisdom about the values of this parameter does not apply.

The matrices $N_{u}$ and $N_{d}$ also exist in the usual flavourpreserving $2 \mathrm{HDMs}$, but there they are diagonal and proportional to the quark mass matrices. In fact, in the type-I 2HDM

\footnotetext{
11 As is plain in equations (26), in our model there is a symmetry between the Yukawa couplings of $\Phi_{1}$ and the ones of $\Phi_{2}$, so that, for any given $t, \tan \beta=t$ is just as (im)possible to achieve as $\tan \beta=1 / t$. This is in contrast to what happens in the usual 2HDMs types I and II.
}

$N_{u}=-\frac{M_{u}}{\tan \beta}, \quad N_{d}=-\frac{M_{d}}{\tan \beta}$,

whereas in the type-II $2 \mathrm{HDM}$

$N_{u}=-\frac{M_{u}}{\tan \beta}, \quad N_{d}=M_{d} \tan \beta$.

Now consider Fig. 2, where we have plotted the values of both $\left|\left(N_{d}\right)_{33}\right| / m_{b}$ and $\left|\left(N_{u}\right)_{33}\right| / m_{t}$ as functions of $\tan \beta$.

The green line shown in plot (a), upon which many blue points are superimposed, corresponds to $\left|\left(N_{d}\right)_{33}\right| / m_{b}=$ $1 / \tan \beta$, that one would obtain if the model behaved, for the bottom quarks, as a type-I 2HDM. The red line would correspond to type-II behaviour, viz. $\left|\left(N_{d}\right)_{33}\right| / m_{b}=\tan \beta$. It appears that, in this model, most regions of parameter space yield either approximate type-I behaviour or approximate type-II behaviour for bottom quarks. Note that, although the blue points appear superimposed on the green and red lines, they are not exactly on them - the type-I and type-II behaviours displayed are approximate and there are deviations from them, which indeed can be large, as we observe in particular for low values of $\tan \beta$. In Fig. $2 \mathrm{~b}$ we observe the same behaviour for top quarks - most points have either $\left|\left(N_{u}\right)_{33}\right| / m_{t} \approx \tan \beta$ or $\left|\left(N_{u}\right)_{33}\right| / m_{t} \approx \cot \beta$.

From Eq. (50) it follows that, in the type I 2HDM,

$\frac{\left(N_{u}\right)_{33} m_{b}}{\left(N_{d}\right)_{33} m_{t}}$

is equal to one, whereas in the type II 2HDM, from Eq. (51),

$-\frac{\left(N_{u}\right)_{33}\left(N_{d}\right)_{33}}{m_{t} m_{b}}$

is equal to one. In Fig. 3 we display the quantities (52) and (53) plotted against each other.

That figure shows that, for most points, our model is more similar to the type-I 2HDM, at least in what concerns giving mass to the third generation, i.e. the top-quark and bottomquark masses originate mostly in the Yukawa couplings to the same scalar doublet. However, there are also many allowed points for which the quantity (52) is not unity; for those points another regularity applies, namely the quantity (53) is very close to zero.

Still, one should remember that in our model there is flavour violation in the Yukawa interactions, and one obtains different results from those in Figs. 2 and 3 for both the $(1,1)$ and $(2,2)$ entries of both $N_{d}$ and $N_{u}$. In fact, the deviations from either type I- or type II-like behaviour for the first and second generations are much more pronounced than what one observes in Fig. 2. But, the corresponding Yukawa couplings being much smaller, that has much less importance for the 


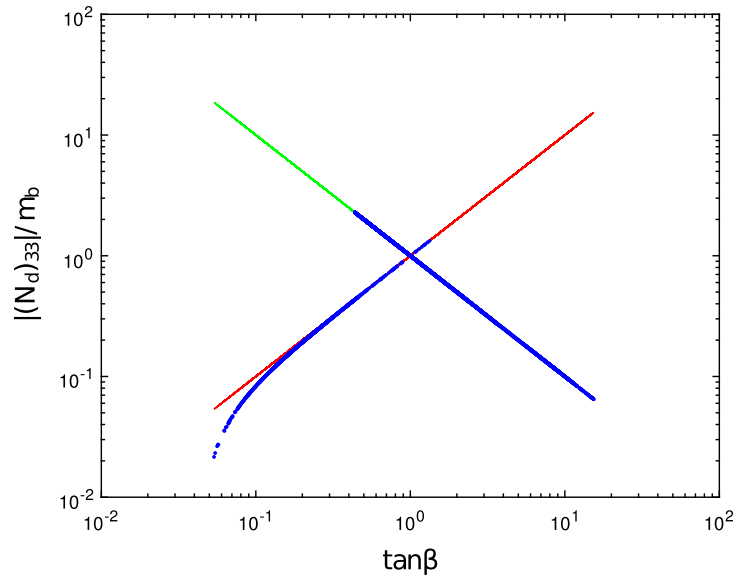

(a)

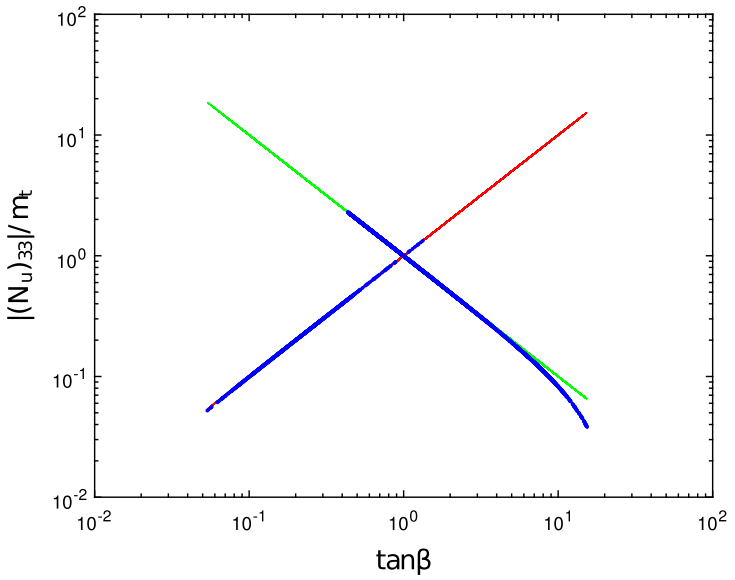

(b)

Fig. 2 a $\left|\left(N_{d}\right)_{33}\right| / m_{b}$ and $\mathbf{b}\left|\left(N_{u}\right)_{33}\right| / m_{t}$ versus $\tan \beta$ for the parameter space points (in blue) which survive all the theoretical and experimental constraints. The green lines correspond to $y=\cot \beta$ and the red lines correspond to $y=\tan \beta$, where $y$ is $\left|\left(N_{d}\right)_{33}\right| / m_{b}$ in $(\mathbf{a})$ and $\left|\left(N_{u}\right)_{33}\right| / m_{t}$ in (b)

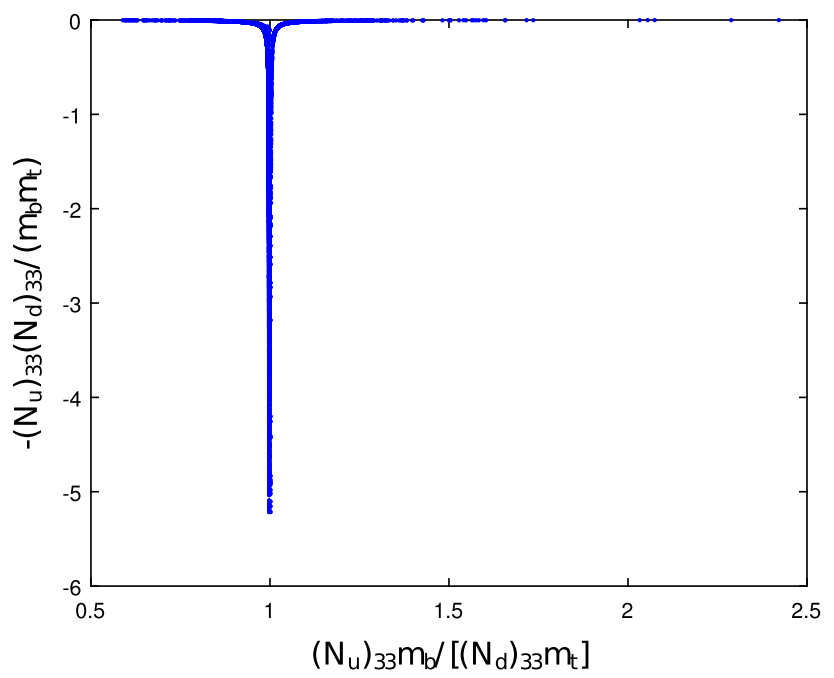

Fig. $3-\left(N_{u}\right)_{33}\left(N_{d}\right)_{33} /\left(m_{b} m_{t}\right)$ versus $\left(N_{u}\right)_{33} m_{b} /\left[\left(N_{d}\right)_{33} m_{t}\right]$

Higgs-boson phenomenology than the third-generation couplings that we have discussed in those figures.

\subsection{Properties of the extra scalars}

We now turn to the extra neutral scalars in the model, $H$ and $A$. The LHC Collaborations have been looking for neutral scalars other than the $125 \mathrm{GeV}$ boson by investigating the production of $W^{+} W^{-}, Z^{0} Z^{0}$, and $\tau \bar{\tau}$, among other channels. The non-observation thus far of meaningful excesses in the cross sections, relatively to their SM expectations, imposes bounds on the masses and couplings of new particles. In our model, the imposition of the top- and meson-physics constraints should force the off-diagonal entries of the matrices $N_{d}$ and $N_{u}$ to be smallish, hence we expect that, just as $h$, the scalars $H$ and $A$ will have reduced flavour-changing interactions. Therefore, our model is expected to behave very much like the flavour-preserving $2 \mathrm{HDMs}$ in what concerns the possibility of evading the current experimental non-observation bounds for the extra scalars. As we will now show, there is a vast parameter space still allowed by the experimental constraints.

We firstly consider the limits coming from the search for resonant $Z^{0} Z^{0}$ pairs by both the ATLAS [19-23] and CMS [24-26] Collaborations. This is a good channel to look for the heavy CP-even scalar $H$, which may decay at tree level as $H \rightarrow Z^{0} Z^{0} .^{12}$ In Fig. 4 we show the points which obey all the constraints described in Sect. 4.1; the points in blue correspond to the requirement that all the $\mu_{X}$ are within $20 \%$ of 1 , and the points in red have all the $\mu_{X}$ less than $10 \%$ away from 1.

The yellow line is the upper $2 \sigma$ bound from the observed limit from Ref. [23]. We observe that most of the allowed parameter space yields a $p p \rightarrow g g \rightarrow H \rightarrow Z^{0} Z^{0}$ cross section below the experimental upper bound; only a few low$m_{H}$ points exceed the bound, but even for those low values of $m_{H}$ there are plenty of points which are still allowed. The tighter constraint of $10 \%$ on the $h$ production rates does not qualitatively change the picture. There is a simple explanation for why low values of the $p p \rightarrow g g \rightarrow H \rightarrow Z^{0} Z^{0}$ event rate should be obtained, namely, in any $C P$-conserving 2HDM (or, indeed, multi-Higgs-doublet model) there is the sum rule

$\left(g_{h Z Z}^{2 \mathrm{HDM}}\right)^{2}+\left(g_{H Z Z}^{2 \mathrm{HDM}}\right)^{2}=\left(g_{h Z Z}^{\mathrm{SM}}\right)^{2}$

\footnotetext{
${ }^{12}$ Unlike the pseudoscalar $A$, which may decay to $Z^{0} Z^{0}$ only through loops.
} 


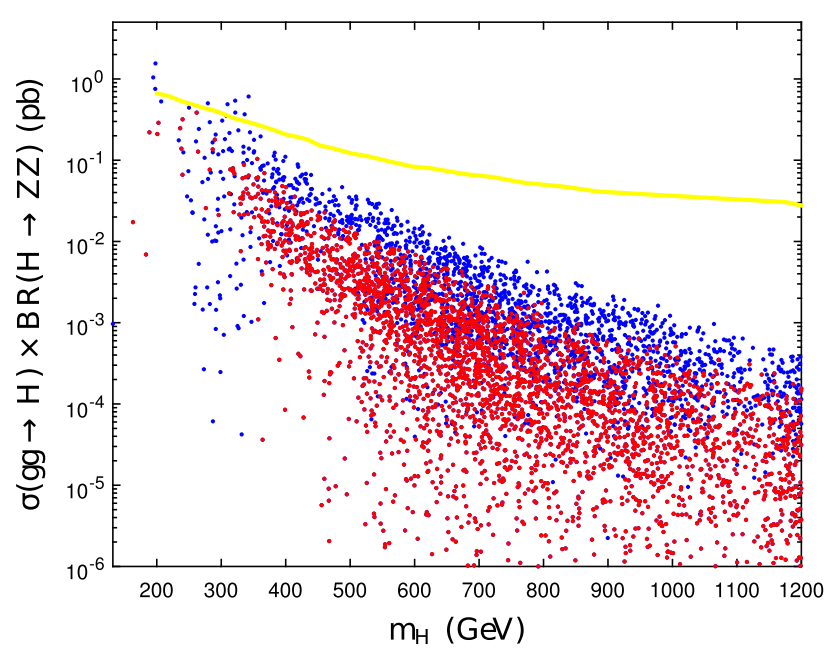

Fig. 4 Cross section of the process $p p \rightarrow g g \rightarrow H \rightarrow Z^{0} Z^{0}$ at $13 \mathrm{TeV}$ collision energy, as a function of the mass of the heavy $C P$ even scalar $H$. All the points displayed obey the constraints for our model described in Sect. 4.1. For the blue points, the $125 \mathrm{GeV}$ scalar $h$ has all its production rates within $20 \%$ of their SM-expected values; for the red points those production rates are all within $10 \%$ of their SM values. The yellow line is the $2 \sigma$ upper bound given in Ref. [23]

for the couplings of the $C P$-even neutral scalars to gaugeboson pairs. Therefore, if the coupling of $h$ to $Z^{0}$ (and $W^{ \pm}$) pairs is very close to its SM value, then the coupling of $H$ to such pairs will be suppressed. Equation (54) is normally expressed through $g_{h Z Z}^{2 \mathrm{HDM}}=s_{\beta-\alpha} g_{h Z \mathrm{SM}}^{\mathrm{SM}}$ and $g_{H Z Z}^{2 \mathrm{HDM}}=$ $c_{\beta-\alpha} g_{h Z Z}^{\mathrm{SM}} ;$ SM-like behaviour of $h$ means $s_{\beta-\alpha} \simeq 1$, which implies $c_{\beta-\alpha} \simeq 0$.

In Fig. 5a we show the gluon-gluon production cross section for a pseudoscalar $A$, multiplied by its branching ratio to a $t \bar{t}$ pair, at LHC. (Similar results were obtained for $H$ instead of $A$, but the obtained values of $\sigma \times$ BR were about one order of magnitude lower than those of $A$ ). There are some LHC results for searches in the $t \bar{t}$ channel $[27,28]$; we have used the results of Ref. [28], although the analysis in that paper does not deal with the 2HDM. In Fig. 5a, the yellow line is the upper $2 \sigma$ bound in figure 11 of Ref. [28]. ${ }^{13}$ The published results only extend down to $m_{A} \simeq 500 \mathrm{GeV}$, but it is clear that no exclusion will occur even for $A$ masses lower than that. As before, the red (blue) points indicate a cut of $10 \%$ (20\%) on the $\mu_{X}$ ratios for the Higgs boson $h$, meant to ensure its SM-like behaviour and compliance with the LHC results. The green points in the same plot are the subset of the red ones for which the width of $A$ is larger than $10 \%$ of its mass: $\Gamma_{A} / m_{A}>0.1$. We have thus far been assuming the validity of the narrow-width approximation and neglecting eventual interferences between backgrounds and signal; by

\footnotetext{
13 That figure concerns the possibility of a spin-2 Kaluza-Klein gravitation excitation, and it is the one for which the lowest values of $\sigma \times \mathrm{BR}$ are achieved, as well as the production channel where the initial state includes two gluons; we have chosen it as a conservative option.
}

marking these large-width points in green, we want to draw attention to the only regions where that approximation might fail. ${ }^{14}$ The conclusion to draw from Fig. $5 \mathrm{a}$ is that the current exclusion bounds from the $t \bar{t}$ resonance searches are easily evaded by our model.

In Fig. $5 b$ we investigate the possibility of the heavy $C P$-even scalar $H$ being observed through its decay to two $125 \mathrm{GeV}$ scalars $h$. This $h h$ channel is being thoroughly studied at the LHC, considering several possible decay channels for both $h$ particles [29-37]; the yellow line in Fig. 5b is the $2 \sigma$ upper bound of figure 6 of Ref. [29]. The blue and red points are the same as before; the green points are the subset of the red ones for which $\Gamma_{H} / m_{H} \geq 0.1$. (Therefore, the green points in Fig. $5 \mathrm{~b}$ do not coincide with the green points in Fig. 5a). Unlike in Fig. 5a, the green points, corresponding to scalars $H$ with a large width, ${ }^{15}$ correspond to smaller values of $\sigma \times \mathrm{BR}$. Just as in the previous figures, we see that virtually all of our parameter space, except a few low-mass points, complies with the existing experimental bounds.

Since the model that we are studying differs from usual versions of the $2 \mathrm{HDM}$ through the existence of FCNC, we have considered the possibility of single-top decays of the heavy (pseudo)scalars $H$ and $A$. Indeed, the non-diagonal Yukawa interactions lead to the possibility of decays like $H \rightarrow t \bar{u}$ and $A \rightarrow c \bar{t}$, which might be observed as top-quark + jet events at the LHC; such events should be quite challenging to study in an hadronic machine such as the LHC, but the recent progress in charmed-jet identification algorithms may be a significant contribution for a future analysis. ${ }^{16}$.

In Fig. 6 we present the expected cross section times branching ratio for both (a) the $H$ and (b) the A. In Fig. 6 we have grouped together all the FCNC decays of the scalars with a single top in the final state, viz. the decays to $t \bar{u}, \bar{t} u$, $t \bar{c}$, and $\bar{t} c$. The top- and meson-physics constraints described in subsection 4.1 usually produce $N_{u}$ matrices with smallish off-diagonal elements, and this yields very small branching ratios for FCNC decays of both $H$ and $A$ - the maximum values that we have obtained were smaller than $5 \times 10^{-4}$, but usual values were much smaller than that. Therefore, the model predicts values for $\sigma \times$ BR usually orders of magnitude below the fentobarn. It is difficult to find experimental bounds on such a search channel, but the search for a $W^{\prime}$ decaying to a single top quark plus a bottom quark [39] can at least give a rough idea of the current sensitivity of the LHC for a top + jet resonance analysis. Though the mass range is different (the analysis of Ref. [39] starts at $1 \mathrm{TeV}$ ), the bounds shown in that paper for the cross section times the branch-

\footnotetext{
${ }^{14}$ Notice, though, that the width of $A$ is never larger than $29.2 \%$ of its mass for the points obtained in our fit.

15 But, for all the points analyzed the width of $H$ was never larger than $35 \%$ of its mass.

16 We thank Nikolaos Rompotis for this comment. See also Ref. [38]
} 


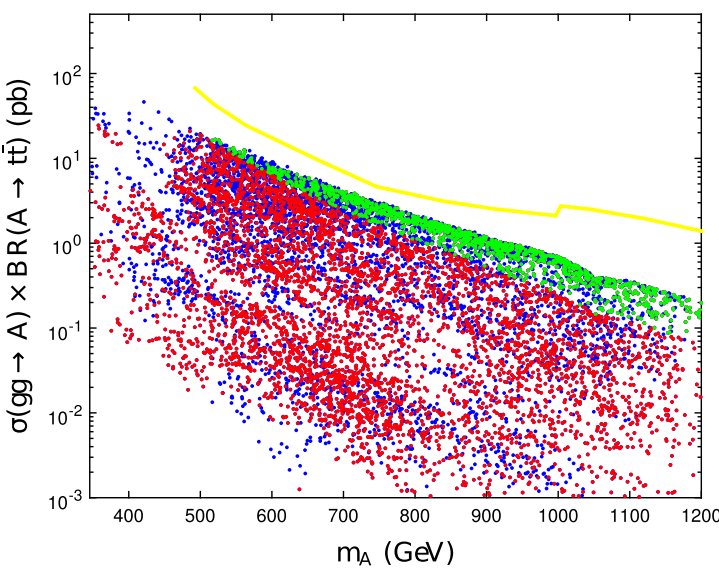

(a)

Fig. 5 a The gluon-gluon production and decay to $t \bar{t}$ of the pseudoscalar $A$ versus its mass. The yellow line is the upper $2 \sigma$ bound in figure 11 of Ref. [28]. b The gluon-gluon production and decay to $h h$ of the scalar $H$ versus its mass. All the points displayed obey the constraints described in Sect. 4. For the blue points, $h$ has production rates

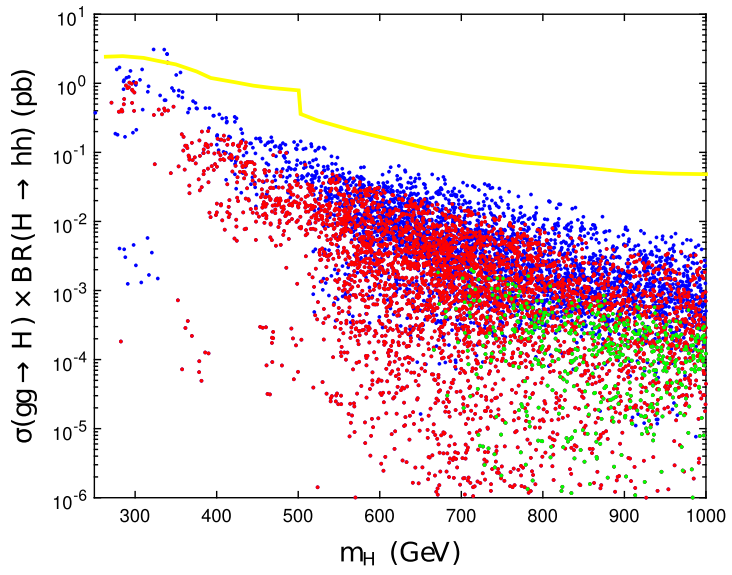

(b)

within $20 \%$ of its SM-expected values; for the red points those production rates are within $10 \%$ of the SM value. The green points are a subset of the red ones, for which the width of the scalar in each plot is larger than $10 \%$ its mass. The yellow line is the $2 \sigma$ upper bound in figure 6 of Ref. [29]

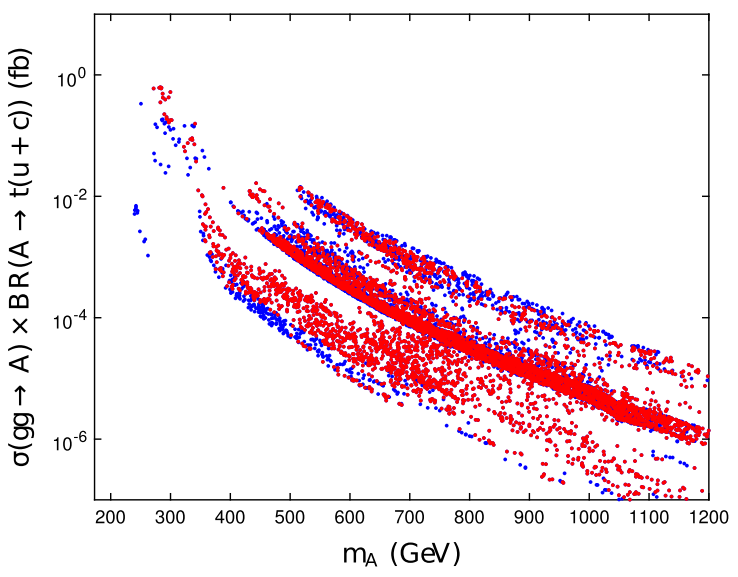

(b)

Fig. 6 a The gluon-gluon production and the decay to $t q$ of a the pseudoscalar $A$ and $\mathbf{b}$ the heavy $C P$-even scalar $H$ versus their respective masses. The colour code is the same as in the previous figures

ing ratio are of order $0.1 \mathrm{pb}$, and therefore much above the predicted $\sigma \times$ BR shown for our model in Fig. 6 .

\section{Conclusions}

In this paper we have presented a two-Higgs-doublet model that attempts a partial solution of the strong $C P$ problem by relegating a possible generation of a nonzero $\theta$ to the twoloop level. Our model achieves this by postulating a soft $C P$ violation that transfers itself just to the CKM matrix, with no $C P$ violation anywhere else in the model, especially no $C P$ violation in scalar-pseudoscalar mixing.
We do not claim that our model achieves a full solution of the strong $C P$ problem, because the $\theta$ generated at twoloop level might still be too large. However, since in our model $C P$ violation exists only in the CKM matrix, one may expect $\theta$ to be proportional to $J \sim 10^{-5}$, the only $C P$ violating invariant quantity in that matrix. Adding in a twoloop factor $\left(16 \pi^{2}\right)^{-2} \sim 10^{-4}$ and [40] probable suppression factors $m_{q} / m_{W}$, where $m_{q}$ is a generic second-generation quark mass, one might well reach a sufficiently small $\theta$.

Of course, a $2 \mathrm{HDM}$ where $C P$ violation only occurs in the CKM matrix eschews one of the motivations for multiHiggs-doublet models, namely, obtaining extra sources of $C P$ violation in order to reach a sufficiently large baryon 
number of the Universe. We have nothing to say about this insufficiency.

We have investigated the compatibility of our model with the outstanding experimental constraints, in particular on the flavour-changing neutral currents. Our model can easily evade them, at the price of cancelations that might be qualified as fine-tuning. We find that the new scalars in our model may in some cases be little heavier than the observed Higgs particle of mass $125 \mathrm{GeV}$. That will not necessarily make them easy to discover, though, as we have seen in Sect. 4.

Acknowledgements PF thanks Miguel Nebot for several enlightening discussions concerning the fit to the meson sector, and Nuno Castro and Nikolaos Rompotis for information concerning LHC experimental constraints. PF is supported in part by the CERN fund grant CERN/FIS-PAR/0002/2017, by the HARMONIA project under contract UMO-2015/18/M/ST2/00518 and by the CFTC-UL strategic project UID/FIS/00618/2019. The work of LL is supported by the Portuguese Fundação para a Ciência e a Tecnologia through the projects PTDC/FIS-PAR/29436/2017, CERN/FIS-PAR/0004/2017, and UID/FIS/00777/2019; those projects are partly funded by POCTI (FEDER), COMPETE, QREN, and the European Union.

Data Availability Statement This manuscript has no associated data or the data will not be deposited. [Authors' comment: This is a theoretical study and no experimental date was listed.]

Open Access This article is distributed under the terms of the Creative Commons Attribution 4.0 International License (http://creativecomm ons.org/licenses/by/4.0/), which permits unrestricted use, distribution, and reproduction in any medium, provided you give appropriate credit to the original author(s) and the source, provide a link to the Creative Commons license, and indicate if changes were made.

Funded by SCOAP $^{3}$.

\section{A The decay $\bar{B} \rightarrow X_{s} \gamma$}

The decays of a bottom-flavoured meson to a strangeflavoured meson and a photon proceed via the quark transition $b \rightarrow s \gamma$. Those decays constitute one of the most relevant constraints on the parameter space of a multi-Higgsdoublet model, because they receive important contributions from loops with charged scalars. This is because the interactions of a charged scalar with down-type quarks may be substantially enhanced by ratios of VEVs. Consequently, in a $2 \mathrm{HDM}$ the constraints from $b \rightarrow s \gamma$ typically eliminate substantial regions of the $m_{H^{+-}} \tan \beta$ plane.

In the model under discussion in this paper, the occurrence of tree-level FCNC means that the neutral scalars also contribute to $b \rightarrow s \gamma$, unlike what happens in flavour-conserving 2HDMs. We follow the general analysis of Ref. [41] to take into account both the charged and the neutral scalars' contributions. We write the Yukawa interactions of our model in the notation

$$
\begin{aligned}
& \mathcal{L}_{\text {physical }} \\
& =\cdots+\sum_{S=h, H, A} S \sum_{k, j=d, s, b} \bar{k}\left(L_{k j}^{S} P_{L}+R_{k j}^{S} P_{R}\right) j \\
& \quad+\left[H^{+} \sum_{\alpha=u, c, t} \bar{\alpha} \sum_{j=b, s, d}\left(L_{\alpha j}^{C} P_{L}+R_{\alpha j}^{C} P_{R}\right) j+\text { H.c. }\right],
\end{aligned}
$$

with coefficients defined as ${ }^{17}$

$$
\begin{aligned}
L_{k j}^{h} & =\frac{s_{\beta-\alpha} m_{k} \delta_{k j}-c_{\beta-\alpha}\left(N_{d}^{*}\right)_{j k}}{v}, \\
R_{k j}^{h} & =\frac{s_{\beta-\alpha} m_{k} \delta_{k j}-c_{\beta-\alpha}\left(N_{d}\right)_{k j}}{v}, \\
L_{k j}^{H} & =\frac{c_{\beta-\alpha} m_{k} \delta_{k j}+s_{\beta-\alpha}\left(N_{d}^{*}\right)_{j k}}{v}, \\
R_{k j}^{H} & =\frac{c_{\beta-\alpha} m_{k} \delta_{k j}+s_{\beta-\alpha}\left(N_{d}\right)_{k j}}{v}, \\
L_{k j}^{A} & =\frac{i\left(N_{d}^{*}\right)_{j k}}{v}, \\
R_{k j}^{A} & =\frac{-i\left(N_{d}\right)_{k j}}{v}, \\
L_{\alpha j}^{C} & =\frac{\sqrt{2}}{v} \sum_{\beta=u, c, t}\left(N_{u}^{*}\right)_{\beta \alpha} V_{\beta j}, \\
R_{\alpha j}^{C} & =-\frac{\sqrt{2}}{v} \sum_{l=d, s, b} V_{\alpha l}\left(N_{d}\right)_{l j} .
\end{aligned}
$$

The Wilson coefficients required for the computation of $b \rightarrow$ $s \gamma$ are [41]

$$
\begin{aligned}
C_{7}^{\prime}(\mu) & =g-\frac{e}{3}, \\
\Delta C_{7}(\mu) & =g^{\prime}-\frac{e^{\prime}}{3}, \\
C_{8}^{\prime}(\mu) & =f+e, \\
\Delta C_{8}(\mu) & =f^{\prime}+e^{\prime},
\end{aligned}
$$

where

$$
\begin{aligned}
f= & -\frac{v^{2}}{4 V_{t s}^{*} V_{t b} m_{H^{+}}^{2}} \sum_{\alpha=u, c, t} R_{\alpha s}^{C *} \\
& \times\left[R_{\alpha b}^{C} I_{3}\left(\frac{m_{\alpha}^{2}}{m_{H^{+}}^{2}}\right)+L_{\alpha b}^{C} \frac{m_{\alpha}}{m_{b}} I_{4}\left(\frac{m_{\alpha}^{2}}{m_{H^{+}}^{2}}\right)\right], \\
g= & -\frac{v^{2}}{4 V_{t s}^{*} V_{t b} m_{H^{+}}^{2}} \sum_{\alpha=u, c, t} R_{\alpha s}^{C *}
\end{aligned}
$$

$\overline{17}$ In our model the matrices $N_{u}$ and $N_{d}$ are real, still we write the coefficients in the general form that follows from Eq. (15), viz. allowing for complex $N_{u}$ and $N_{d}$. 


$$
\begin{aligned}
\times & {\left[R_{\alpha b}^{C} I_{5}\left(\frac{m_{\alpha}^{2}}{m_{H^{+}}^{2}}\right)+L_{\alpha b}^{C} \frac{m_{\alpha}}{m_{b}} I_{6}\left(\frac{m_{\alpha}^{2}}{m_{H^{+}}^{2}}\right)\right], } \\
e= & -\frac{v^{2}}{4 V_{t s}^{*} V_{t b}} \sum_{S=h, H, A} \frac{1}{m_{S}^{2}} \sum_{k=d, s, b} R_{k s}^{S *} \\
& \times\left[R_{k b}^{S} I_{3}\left(\frac{m_{k}^{2}}{m_{S}^{2}}\right)+L_{k b}^{S} \frac{m_{k}}{m_{b}} I_{4}\left(\frac{m_{k}^{2}}{m_{S}^{2}}\right)\right],
\end{aligned}
$$

and

$$
\begin{aligned}
f^{\prime} & =f\left(R^{C} \leftrightarrow L^{C}\right), \\
g^{\prime} & =g\left(R^{C} \leftrightarrow L^{C}\right), \\
e^{\prime} & =e\left(R^{S} \leftrightarrow L^{S}\right) \text { for } S=h, H, A .
\end{aligned}
$$

The functions $I_{3,4,5,6}$ are given in equations (40)-(43) of Ref. [41].

To compute the overall branching ratio of $b \rightarrow s \gamma$ we follow refs. $[42,43]$. We use the effective operators described above, defined as being at the Fermi scale $\mu=m_{W}$, and include NLO QCD corrections by choosing $m_{b}$ as the renormalization scale. Let $\eta=\alpha_{S}\left(m_{W}\right) / \alpha_{S}\left(m_{b}\right)=0.5651$ [42] be the ratio of the running strong coupling constant between scales $m_{W}$ and $m_{b}$. We compute

$$
\begin{aligned}
\Delta C\left(m_{b}\right)= & \eta^{16 / 23} \Delta C_{7}\left(m_{W}\right) \\
& +\frac{8}{3}\left(\eta^{14 / 23}-\eta^{16 / 23}\right) \Delta C_{8}\left(m_{W}\right), \\
C^{\prime}\left(m_{b}\right)= & \eta^{16 / 23} C_{7}^{\prime}\left(m_{W}\right) \\
& +\frac{8}{3}\left(\eta^{14 / 23}-\eta^{16 / 23}\right) C_{8}^{\prime}\left(m_{W}\right),
\end{aligned}
$$

and then [44]

$$
\begin{aligned}
\mathrm{BR}(b \rightarrow s \gamma)= & \mathrm{BR}(b \rightarrow s \gamma)_{\mathrm{SM}} \\
& +\left(2.47 \times 10^{-3}\right)\left\{\left|\Delta C\left(m_{b}\right)\right|^{2}+\left|C^{\prime}\left(m_{b}\right)\right|^{2}\right. \\
& \left.-0.706 \operatorname{Re}\left[\Delta C\left(m_{b}\right)\right]\right\},
\end{aligned}
$$

where $\mathrm{BR}(b \rightarrow s \gamma)_{\mathrm{SM}}=3.15 \times 10^{-4}$. We consider our model to be in compliance with the $b \rightarrow s \gamma$ data if it yields a branching ratio within twice the experimental error bar, viz. we require $2.4406 \times 10^{-4}<\mathrm{BR}(b \rightarrow s \gamma)<3.8594 \times$ $10^{-4}$.

\section{B The neutral meson-antimeson observables}

The FCNC induced by the off-diagonal entries of $N_{d}$ and $N_{u}$ lead to tree-level contributions to flavour observables such as $C P$ violation through the parameter $\epsilon_{K}$ and the mass differences in the $K^{0}, B_{d}^{0}, B_{s}^{0}$, and $D^{0}$ meson-antimeson systems. These are sensitive observables and new-physics contributions to them may easily be overwhelming. Thus, we must make sure that the contributions to them from the scalar sec- tor of our model conform to the current data. We use the numbers listed in Ref. [11].

B.1 $K^{0}-\bar{K}^{0}$ observables

Two $K^{0}$ meson observables are sensitive to the tree-level FCNC contributions from the scalar sector: the $C P$-violating parameter $\epsilon_{K}$ and the mass difference between $K_{S}$ and $K_{L}$. Both observables arise from the matrix element effecting the trasition $\bar{K}^{0} \rightarrow K^{0}$, called $M_{21}$. This receives contributions from the SM, via box diagrams, and from new physics (NP), through FCNC in the scalar sector: $M_{21}=M_{21}^{\mathrm{SM}}+M_{21}^{\mathrm{NP}}$. We use the results presented in Ref. [45]. The SM contribution originates in a box diagram and is given by

$$
\begin{aligned}
M_{21}^{\mathrm{SM}}= & -\frac{G_{F}^{2} m_{W}^{2} f_{K}^{2} m_{K} B_{K}}{12 \pi^{2}}\left[\eta_{1} \lambda_{c}^{2} S_{0}\left(x_{c}\right)+\eta_{2} \lambda_{t}^{2} S_{0}\left(x_{t}\right)\right. \\
& \left.+2 \eta_{3} \lambda_{c} \lambda_{t} S_{0}\left(x_{c}, x_{t}\right)\right]
\end{aligned}
$$

where $G_{F}$ is the Fermi constant, $m_{W}$ is the $W$-boson mass, $f_{K}=0.1555 \mathrm{GeV}$ is the $K$-meson decay constant, $m_{K}=$ $0.497611 \mathrm{GeV}$ is the $K$-meson mass, and $B_{K}=0.723$ parameterizes the error in the vacuum-insertion approximation for the relevant matrix element. The $x_{q}=\left(m_{q} / m_{W}\right)^{2}$ and $\lambda_{q}=V_{q d}^{*} V_{q s}$ for $q=c, t$. The functions $S_{0}$ are given in equations (B.15) and (B.16) of Ref. [45]. Finally, the parameters $\eta_{1}=1.38, \eta_{2}=0.57$, and $\eta_{3}=0.47$ account for QCD corrections.

The new-physics contribution originates in the tree-level exchange of $h, H$, and $A$. One has, by using the vacuuminsertion approximation for the matrix elements of the operators, ${ }^{18}$

$$
\begin{aligned}
M_{21}^{\mathrm{NP}}= & \frac{f_{K}^{2} m_{K}}{96 v^{2}}\left\{\left[\left(N_{d}^{*}\right)_{d s}^{2}+\left(N_{d}\right)_{s d}^{2}\right]\right. \\
& \times \frac{10 m_{K}^{2}}{\left(m_{s}+m_{d}\right)^{2}}\left(\frac{1}{m_{A}^{2}}-\frac{c_{\beta-\alpha}^{2}}{m_{h}^{2}}-\frac{s_{\beta-\alpha}^{2}}{m_{H}^{2}}\right) \\
& +4\left(N_{d}^{*}\right)_{d s}\left(N_{d}\right)_{s d}\left[1+\frac{6 m_{K}^{2}}{\left(m_{s}+m_{d}\right)^{2}}\right] \\
& \left.\times\left(\frac{1}{m_{A}^{2}}+\frac{c_{\beta-\alpha}^{2}}{m_{h}^{2}}+\frac{s_{\beta-\alpha}^{2}}{m_{H}^{2}}\right)\right\}
\end{aligned}
$$

Notice that $M_{21}^{\mathrm{NP}}$ in our model is real.

The $K_{S}-K_{L}$ mass difference is given by $\Delta m_{K}=2\left|M_{21}\right|$. Unfortunately, the SM contribution to $\Delta m_{K}$ is affected by considerable uncertainties, stemming from long-distance, difficult to compute contributions to $M_{21}^{\mathrm{SM}}$, and also from imprecisions in the value of $B_{K}$. Therefore, we just require that the new-physics term does not give a contribution to $\Delta m_{K}$ larger than the experimental value, i.e. while fitting

\footnotetext{
${ }^{18}$ See refs. [45] and [8] for a detailed derivation of Eq. (63).
} 
the parameters of the model we demand that $2\left|M_{21}^{\mathrm{NP}}\right|<$ $3.484 \times 10^{-15} \mathrm{GeV}$.

It is expected that the uncertainties which trouble the calculation of $\Delta m_{K}$ do not affect the computation of $\epsilon_{K}$, given by

$\epsilon_{K}=2.228 \times 10^{-3}=-\frac{\operatorname{Im}\left(M_{21} \lambda_{u}^{* 2}\right)}{\sqrt{2} \Delta m_{K}\left|\lambda_{u}\right|^{2}}$.

In Eq. (64), we use in the numerator $M_{21}=M_{21}^{\mathrm{SM}}+M_{21}^{\mathrm{NP}}$, while for $\Delta m_{K}$ in the denominator we use the experimental value. We accept fit results which give $\epsilon_{K}$ within a $10 \%$ deviation from the central value.

\section{B.2 $B_{H}-B_{L}$ mass differences}

The mass differences in the meson-antimeson $B_{d}^{0}-\bar{B}_{d}^{0}$ and $B_{s}^{0}-\bar{B}_{s}^{0}$ systems are well measured and their theoretical calculation, unlike that of $\Delta m_{K}$, is reliable. We use, for the $\bar{B}_{d}^{0} \rightarrow B_{d}^{0}$ transition,

$M_{21}^{\mathrm{SM}}=-\frac{G_{F}^{2} m_{W}^{2} f_{B_{d}}^{2} m_{B_{d}} B_{B_{d}}}{12 \pi^{2}} \eta_{B_{d}}\left(V_{t b} V_{t d}^{*}\right)^{2} S_{0}\left(x_{t}\right)$,

with $f_{B_{d}}=0.1902 \mathrm{GeV}, m_{B_{d}}=5.280 \mathrm{GeV}, B_{B_{d}}=1.219$, and $\eta_{B_{d}}=0.55$. Note that in Eq. (65) one uses only the box diagram with top-quark internal lines. The NP contribution is given by an expression analogous to Eq. (63), with the obvious substitutions $f_{K} \rightarrow f_{B_{d}}, m_{K} \rightarrow m_{B_{d}}$, and $m_{s} \rightarrow$ $m_{b}$. We accept the result of the fit if $2\left|M_{21}^{\mathrm{SM}}+M_{21}^{\mathrm{NP}}\right|$ is within $10 \%$ of the experimental value $\Delta m_{B_{d}}=3.333 \times 10^{-13} \mathrm{GeV}$.

For the $\bar{B}_{s}^{0} \rightarrow B_{s}^{0}$ transition we have Eq. (65) with all indices $d \rightarrow s$ and $f_{B_{s}}=0.228 \mathrm{GeV}, m_{B_{s}}=5.367 \mathrm{GeV}$, $B_{B_{s}}=1.28$, and $\eta_{B_{s}}=0.55$. The NP contribution is given by an expression analogous to Eq. (63), with the obvious substitutions $f_{K} \rightarrow f_{B_{s}}, m_{K} \rightarrow m_{B_{s}}$, and $m_{d} \rightarrow m_{b}$. We accept the result of the fit if $2\left|M_{21}^{\mathrm{SM}}+M_{21}^{\mathrm{NP}}\right|$ is within $10 \%$ of the experimental value $\Delta m_{B_{s}}=1.17 \times 10^{-11} \mathrm{GeV}$.

\section{B.3 The mass difference in the $D^{0}-\bar{D}^{0}$ system}

There are also contributions to the mass difference in the meson system $D^{0}-\bar{D}^{0}$. As in the $K^{0}-\bar{K}^{0}$ system, there are considerable uncertainties in the calculation of $M_{21}^{\mathrm{SM}}$. Therefore, once again, we resort to requiring only the New Physics contribution not to be too large. We have

$$
\begin{aligned}
M_{21}^{\mathrm{NP}}= & \frac{f_{D}^{2} m_{D}}{96 v^{2}}\left\{\left[\left(N_{u}^{*}\right)_{u c}^{2}+\left(N_{u}\right)_{c u}^{2}\right]\right. \\
& \times \frac{10 m_{D}^{2}}{\left(m_{c}+m_{u}\right)^{2}}\left(\frac{1}{m_{A}^{2}}-\frac{c_{\beta-\alpha}^{2}}{m_{h}^{2}}-\frac{s_{\beta-\alpha}^{2}}{m_{H}^{2}}\right)
\end{aligned}
$$

$$
\begin{aligned}
& +4\left(N_{u}^{*}\right)_{c u}\left(N_{u}\right)_{u c}\left[1+\frac{6 m_{D}^{2}}{\left(m_{c}+m_{u}\right)^{2}}\right] \\
& \left.\times\left(\frac{1}{m_{A}^{2}}+\frac{c_{\beta-\alpha}^{2}}{m_{h}^{2}}+\frac{s_{\beta-\alpha}^{2}}{m_{H}^{2}}\right)\right\},
\end{aligned}
$$

with $f_{D}=0.212 \mathrm{GeV}$ and $m_{D}=1.865 \mathrm{GeV}$. Conservatively, we require $2\left|M_{21}^{\mathrm{NP}}\right|$ to be smaller than the measured mass difference $6.253 \times 10^{-15} \mathrm{GeV}$.

\section{C $Z \rightarrow b \bar{b}$ constraints}

A potentially very important constraint to two-Higgs-doublet models (2HDMs) stems from the measurement of the decay $Z \rightarrow b \bar{b}$. We follow the treatment of that decay in refs. [4648]. The Lagrangian for the $Z b \bar{b}$ vertex is written as

$\mathcal{L}_{Z b \bar{b}}=-\frac{e Z_{\mu}}{s_{W} c_{W}} \bar{b} \gamma^{\mu}\left(\bar{g}_{b}^{L} P_{L}+\bar{g}_{b}^{R} P_{R}\right) b$,

where the coefficients $\bar{g}_{b}^{L, R}$ are, at tree level in the SM, $\bar{g}_{b}^{L}=-1 / 2+s_{W}^{2} / 3$ and $\bar{g}_{b}^{R}=s_{W}^{2} / 3$. In both the SM and in extensions thereof, these coefficients get one-loop contributions. To wit, in the $2 \mathrm{HDM}$ the contributions of loops with charged scalars to $\bar{g}_{b}^{L}$ and $\bar{g}_{b}^{R}$ are given by

$\delta \bar{g}_{b}^{L}=\frac{\sqrt{2} G_{F}}{16 \pi^{2}}\left|\left(N_{u}^{\dagger} V\right)_{33}\right|^{2} f_{1}\left(\frac{m_{t}^{2}}{m_{H^{+}}^{2}}\right)$,

$\delta \bar{g}_{b}^{R}=-\frac{\sqrt{2} G_{F}}{16 \pi^{2}}\left|\left(V N_{d}\right)_{33}\right|^{2} f_{1}\left(\frac{m_{t}^{2}}{m_{H^{+}}^{2}}\right)$,

where

$f_{1}(x)=\frac{x}{x-1}\left(1-\frac{\ln x}{x-1}\right)$.

The contributions of loops with neutral scalars are expected to be small, both for 2HDMs with flavour conservation [4648] or without it [49]; we neglect them. In order to take into account the current experimental results on the observable quantities $R_{b}$ and $A_{b}$ (see refs. $[11,46]$ ), we have required that the charged-scalar contribution added to the SM one, viz. $\bar{g}_{b}^{L}=-0.42112+\delta \bar{g}_{b}^{L}$ and $\bar{g}_{b}^{R}=0.07744+\delta \bar{g}_{b}^{R}$, does not deviate from the SM prediction by more than $2 \sigma$, viz. $2\left(\bar{g}_{b}^{L}\right)^{2}+2\left(\bar{g}_{b}^{R}\right)^{2}=0.36782 \pm 0.00143$.

\section{A benchmark point}

To illustrate the model, we provide a specific point, which is meant to serve only as an example. The input in the scalar 
sector is

$$
\begin{aligned}
& v=\sqrt{v_{1}^{2}+v_{2}^{2}}=246 \mathrm{GeV}, \quad v_{1}=145.48 \mathrm{GeV}, \\
& \beta-\alpha=289.46^{\circ}, \\
& m_{h}=125 \mathrm{GeV}, \quad m_{H}=688.46 \mathrm{GeV}, \\
& m_{A}=364.01 \mathrm{GeV}, \quad m_{H^{+}}=712.00 \mathrm{GeV} .
\end{aligned}
$$

To this input correspond the following (approximate) values for the parameters of the scalar potential:

$$
\begin{aligned}
& \mu_{1}=89682.55 \mathrm{GeV}^{2}, \quad \mu_{2}=31942.59 \mathrm{GeV}^{2}, \\
& \mu_{3}=63188.85 \mathrm{GeV}^{2}, \\
& \lambda_{1}=9.9708, \quad \lambda_{2}=3.7121, \quad \lambda_{3}=6.8336, \\
& \lambda_{4}=-12.3750 .
\end{aligned}
$$

It is easy to confirm that the values (71) fulfil all the conditions $(23,24,25)$. Notice in Eq. (70b) that the masses of all four scalars are neither too close nor too far away from each other.

The Yukawa-coupling matrices are as in Eq. (26), with

$$
\begin{aligned}
& b_{1}=1.0761 \times 10^{-3}, \quad d_{1}=1.9555 \times 10^{-4}, \\
& f_{1}=5.1710 \times 10^{-5}, \\
& b_{2}=-9.3709 \times 10^{-4}, \quad d_{2}=-3.0026 \times 10^{-2}, \\
& f_{2}=0, \\
& p_{1}=6.9338 \times 10^{-2}, \quad q_{1}=-3.0282 \times 10^{-4}, \\
& r_{1}=-1.3664 \times 10^{-2}, \quad{ }^{-4}=0.2531 \times 10^{-3} .
\end{aligned}
$$

We also input $\aleph_{1}=0, \aleph_{2}=1.33 \mathrm{rad}$. One thus obtains quark masses and a CKM matrix in agreement with Eqs. $(44,45)$. The matrices that parameterize the FCNC are

$$
\begin{aligned}
& N_{d}=\left(\begin{array}{ccc}
7.4 \times 10^{-3} & 2.7 \times 10^{-2} & 4.2 \times 10^{-2} \\
-1.8 \times 10^{-5} & 0.15 & -5.0 \times 10^{-3} \\
5.3 \times 10^{-5} & -3.9 \times 10^{-7} & -3.09
\end{array}\right), \\
& N_{u}=\left(\begin{array}{ccc}
1.2 \times 10^{-2} & -0.29 & -1.61 \\
6.4 \times 10^{-2} & -0.93 & -14.9 \\
-2.7 \times 10^{-3} & 1.3 \times 10^{-4} & -126
\end{array}\right)
\end{aligned}
$$

One sees that some off-diagonal matrix elements of $N_{d}$ are not very small, and some off-diagonal matrix elements of $N_{u}$ - which is almost a triangular matrix - are pretty large. There is a cancelation of about one part in 47 between the contributions to the neutral- $D$-meson mass difference of the neutral scalars $h$ and $H$ on the one hand and of the pseudoscalar $A$ on the other hand; there are analogous, yet milder, cancelations among the contributions to the other neutralmeson mass differences. In general, we have found that the $D$-meson mass difference constraint requires quite strongly fine-tuned cancelations when the neutral scalars have low masses, while the constraints from all other neutral-meson systems are much easier to satisfy and mostly require no fine-tuning. Still, notice that this benchmark point has one particle (the pseudoscalar $A$ ) with relatively low mass.

With this benchmark point, the coupling modifiers defined in Eqs. (46) and (48) are

$\kappa_{V}=0.9993, \quad \kappa_{t}=0.9721, \quad \kappa_{b}=0.9720, \quad \kappa_{\tau}=1.0502$,

and some of the phenomenological quantities computed in Sect. 4 are found to be:

$$
\begin{aligned}
\operatorname{BR}\left(h \rightarrow q \bar{q}^{\prime}\right) & =6.89 \times 10^{-11}, \\
\sigma(g g \rightarrow h) & =38.98 \mathrm{pb}, \\
\sigma\left(g g \rightarrow H \rightarrow Z^{0} Z^{0}\right) & =0.75 \mathrm{fb}, \\
\sigma\left(g g \rightarrow A \rightarrow t \bar{q}_{u}\right) & =0.03 \mathrm{fb},
\end{aligned}
$$

where (1) $q \bar{q}^{\prime}$ refers to a sum over all possible FCNC decays of $h,(2)$ the cross sections are for a LHC center-of-mass collision of $13 \mathrm{TeV}$, (3) the FCNC decays of $A$ involve $q_{u}=u$ and $q_{u}=c$, and (4) we have grouped together all the FCNC decays of the scalars with a single top in the final state.

\section{References}

1. J. Dragos, T. Luu, A. Shindler, J. de Vries, A. Yousif, Confirming the existence of the strong $\mathrm{CP}$ problem in lattice QCD with the gradient flow. arXiv:1902.03254 [hep-lat]

2. A. Iwazaki, Nonvanishing pion masses for vanishing bare quark masses. arXiv:1901.03045 [hep-ph]

3. R.D. Peccei, H.R. Quinn, C.P. Conservation, in the presence of pseudoparticles, Phys. Rev. Lett. 38, 1440; Constraints imposed by $C P$ conservation in the presence of pseudoparticles. Phys. Rev. D 16(1977), 1791 (1977)

4. W.A. Bardeen, Instanton-triggered chiral symmetry breaking, the $U$ (1) problem, and a possible solution to the strong CP problem. arXiv:1812.06041 [hep-ph]

5. A.L. Cherchiglia, C.C. Nishi, Solving the strong CP problem with non-conventional CP. JHEP 1903, 040 (2019)

6. G.C. Branco, P.M. Ferreira, L. Lavoura, M.N. Rebelo, M. Sher, J.P. Silva, Theory and phenomenology of two-Higgs-doublet models. Phys. Rept. 516, 1 (2012). arXiv:1106.0034 [hep-ph]

7. G. Segrè, H.A. Weldon, Natural suppression of strong $P$ - and $T$ invariance violations and calculable mixing angles in $\mathrm{SU}(2) \otimes \mathrm{U}(1)$. Phys. Rev. Lett. 42, 1191 (1979)

8. P.M. Ferreira, L. Lavoura, J.P. Silva, A soft origin for CKM-type CP violation. Phys. Lett. B 704, 179 (2011). arXiv:1102.0784 [hep-ph]

9. A. Barroso, P.M. Ferreira, I.P. Ivanov, R. Santos, J.P. Silva, Evading death by vacuum. Eur. Phys. J. C 73, 2537 (2013). arXiv:1211.6119 [hep-ph]

10. I.P. Ivanov, J.P. Silva, Tree-level metastability bounds for the most general two Higgs doublet model. Phys. Rev. D 92, 055017 (2015). arXiv:1507.05100 [hep-ph]

11. M. Tanabashi et al., [Particle Data Group], Review of Particle Physics. Phys. Rev. D 98, 030001 (2018)

12. D. Toussaint, Renormalization effects from superheavy Higgs particles. Phys. Rev. D 18, 1626 (1978) 
13. S. Kanemura, Y. Okada, H. Taniguchi, K. Tsumura, Indirect bounds on heavy scalar masses of the two-Higgs-doublet model in light of recent Higgs boson searches. Phys. Lett. B 704, 303 (2011). arXiv:1108.3297 [hep-ph]

14. P.M. Ferreira, J.F. Gunion, H.E. Haber, R. Santos, Probing wrongsign Yukawa couplings at the LHC and a future linear collider. Phys. Rev. D 89, 115003 (2014). arXiv:1403.4736 [hep-ph]

15. P.M. Ferreira, R. Guedes, M.O.P. Sampaio, R. Santos, Wrong sign and symmetric limits and non-decoupling in 2HDMs. JHEP 1412, 067 (2014). arXiv:1409.6723 [hep-ph]

16. R.V. Harlander, S. Liebler, H. Mantler, SusHi: a program for the calculation of Higgs production in gluon fusion and bottom-quark annihilation in the Standard Model and the MSSM. Comput. Phys. Commun. 184, 1605 (2013). arXiv:1212.3249 [hep-ph]

17. R.V. Harlander, S. Liebler, H. Mantler, SusHi Bento: beyond NNLO and the heavy-top limit. Comput. Phys. Commun. 212, 239 (2017). arXiv:1605.03190 [hep-ph]

18. M. Nebot, J.P. Silva, Self-cancellation of a scalar in neutral meson mixing and implications for the LHC. Phys. Rev. D 92, 085010 (2015). arXiv:1507.07941 [hep-ph]

19. M. Aaboud et al., [ATLAS Collaboration], Search for $W W / W Z$ resonance production in $\ell v q q$ final states in $p p$ collisions at $\sqrt{s}=13 \mathrm{TeV}$ with the ATLAS detector. JHEP 1803, 042 (2018). arXiv: 1710.07235 [hep-ex]

20. M. Aaboud et al., [ATLAS Collaboration], Searches for heavy $Z Z$ and $Z W$ resonances in the $\ell \ell q q$ and $v v q q$ final states in $p p$ collisions at $\sqrt{s}=13 \mathrm{TeV}$ with the ATLAS detector. JHEP 1803, 009 (2018). arXiv:1708.09638 [hep-ex]

21. M. Aaboud et al., [ATLAS Collaboration], Search for heavy resonances decaying into $W W$ in the $e v \mu \nu$ final state in $p p$ collisions at $\sqrt{s}=13 \mathrm{TeV}$ with the ATLAS detector. Eur. Phys. J. C 78, 24 (2018). arXiv:1710.01123 [hep-ex]

22. ATLAS Collaboration, Study of the Higgs boson properties and search for high-mass scalar resonances in the $H \rightarrow Z Z^{*} \rightarrow 4 \ell$ decay channel at $\sqrt{s}=13 \mathrm{TeV}$ with the ATLAS detector, ATLASCONF-2016-079

23. ATLAS Collaboration, Search for heavy $Z Z$ resonances in the $\ell^{+} \ell^{-} \ell^{+} \ell^{-}$and $\ell^{+} \ell^{-} v \bar{v}$ final states using proton-proton collisions at $\sqrt{s}=13 \mathrm{TeV}$ with the ATLAS detector, ATLAS-CONF-2017058

24. CMS Collaboration, Search for a heavy scalar boson decaying into a pair of $Z$ bosons in the $2 \ell 2 v$ final state, CMS-PAS-HIG-16-001

25. CMS Collaboration, Measurements of properties of the Higgs boson and search for an additional resonance in the four-lepton final state at $\sqrt{s}=13 \mathrm{TeV}$, CMS-PAS-HIG-16-033

26. CMS Collaboration, Search for new diboson resonances in the dilepton + jets final state at $\sqrt{s}=13 \mathrm{TeV}$ with 2016 data, CMSPAS-HIG-16-034

27. M. Aaboud et al., [ATLAS Collaboration], Search for heavy Higgs Bosons $A / H$ decaying to a top quark pair in $p p$ collisions at $\sqrt{s}=$ $8 \mathrm{TeV}$ with the ATLAS detector. Phys. Rev. Lett. 119, 191803 (2017). arXiv:1707.06025 [hep-ex]

28. M. Aaboud et al. [ATLAS Collaboration], Search for heavy particles decaying into a top-quark pair in the fully hadronic final state in $p p$ collisions at $\sqrt{s}=13 \mathrm{TeV}$ with the ATLAS detector. Phys. Rev. D 99092004 (2019)

29. G. Aad et al., [ATLAS Collaboration], Searches for Higgs boson pair production in the $h h \rightarrow b b \tau \tau, \gamma \gamma W W^{*}, \gamma \gamma b b, b b b b$ channels with the ATLAS detector. Phys. Rev. D 92, 092004 (2015). arXiv:1509.04670 [hep-ex]

30. ATLAS Collaboration, Search for Higgs boson pair production in the $b \bar{b} \gamma \gamma$ final state using $p p$ collision data at $\sqrt{s}=13 \mathrm{TeV}$ with the ATLAS detector, ATLAS-CONF-2016-004
31. CMS Collaboration, Search for Higgs boson pair production in the final state containing two photons and two bottom quarks in proton-proton collisions at $\sqrt{s}=13 \mathrm{TeV}, \mathrm{CMS}-\mathrm{PAS}-\mathrm{HIG}-17-008$

32. M. Aaboud et al., [ATLAS Collaboration], Search for pair production of Higgs bosons in the $b \bar{b} b \bar{b}$ final state using proton-proton collisions at $\sqrt{s}=13 \mathrm{TeV}$ with the ATLAS detector. Phys. Rev. D 94, 052002 (2016). arXiv:1606.04782 [hep-ex]

33. CMS Collaboration, Search for heavy resonances decaying to a pair of Higgs bosons in the four $b$ quark final state in proton-proton collisions at $\sqrt{s}=13 \mathrm{TeV}$, CMS-PAS-B2G-16-026

34. ATLAS Collaboration, Search for pair production of Higgs bosons in the $b \bar{b} b \bar{b}$ final state using proton-proton collisions at $\sqrt{s}=$ $13 \mathrm{TeV}$ with the ATLAS detector, ATLAS-CONF-2016-049

35. ATLAS Collaboration, Search for Higgs boson pair production in the final state of $\gamma \gamma W W^{*}(\rightarrow l \nu j j)$ using $13.3 \mathrm{fb}^{-1}$ of $p p$ collision data recorded at $\sqrt{s}=13 \mathrm{TeV}$ with the ATLAS detector, ATLASCONF-2016-071

36. A.M. Sirunyan et al., [CMS Collaboration], Search for resonant and nonresonant Higgs boson pair production in the $b \bar{b} \ell \nu \ell v$ final state in proton-proton collisions at $\sqrt{s}=13 \mathrm{TeV}$. JHEP 1801, 054 (2018). [arXiv:1708.04188 [hep-ex]]

37. A.M. Sirunyan et al., [CMS Collaboration], Search for Higgs boson pair production in events with two bottom quarks and two tau leptons in proton-proton collisions at $\sqrt{s}=13 \mathrm{TeV}$. Phys. Lett. B 778, 101 (2018). arXiv:1707.02909 [hep-ex]

38. W. Altmannshofer, B. Maddock, D. Tuckler, Rare top decays as probes of flavorful Higgs bosons (2019). arXiv:1904.10956 [hep$\mathrm{ph}]$

39. M. Aaboud et al., [ATLAS Collaboration], Search for $W^{\prime} \rightarrow t b$ decays in the hadronic final state using $p p$ collisions at $\sqrt{s}=$ $13 \mathrm{TeV}$ with the ATLAS detector. Phys. Lett. B 781, 327 (2018). arXiv:1801.07893 [hep-ex]

40. J.R. Ellis, M.K. Gaillard, Strong and weak $C P$ violation. Nucl. Phys. B 150, 141 (1979)

41. L. Lavoura, General formulae for $f_{1} \rightarrow f_{2} \gamma$. Eur. Phys. J. C 29, 191 (2003). arXiv:hep-ph/0302221

42. A.J. Buras, M. Misiak, M. Münz, S. Pokorski, Theoretical uncertainties and phenomenological aspects of $B \rightarrow X_{s} \gamma$ decay, Nucl. Phys. B 424, 374 (1994). arXiv:hep-ph/9311345

43. M. Misiak, M. Steinhauser, NNLO QCD corrections to the $\bar{B} \rightarrow$ $X_{s} \gamma$ matrix elements using interpolation in $m_{c}$. Nucl. Phys. B 764, 62 (2007). arXiv:hep-ph/0609241

44. M. Blanke, B. Shakya, P. Tanedo, Y. Tsai, The birds and the $B$ s in RS: the $b \rightarrow s \gamma$ penguin in a warped extra dimension. JHEP 1208, 038 (2012). arXiv:1203.6650 [hep-ph]

45. G.C. Branco, L. Lavoura, J.P. Silva, CP Violation (Oxford University Press, Oxford, 1999)

46. H.E. Haber, H.E. Logan, Radiative corrections to the $Z b \bar{b}$ vertex and constraints on extended Higgs sectors. Phys. Rev. D 62, 015011 (2000). arXiv:hep-ph/9909335

47. O. Deschamps, S. Monteil, V. Niess, S. Descotes-Genon, S. T'Jampens, V. Tisserand, Two Higgs Doublet of type II facing flavor physics data. Phys. Rev. D 82, 073012 (2010). arXiv:0907.5135 [hep-ph]

48. G. Degrassi, P. Slavich, QCD corrections in two-Higgs-doublet extensions of the standard model with minimal flavor violation. Phys. Rev. D 81, 075001 (2010). arXiv:1002.1071 [hep-ph]

49. J. Hernandez-Sánchez, S. Moretti, R. Noriega-Papaqui, A. Rosado, Off-diagonal terms in Yukawa textures of the type-III 2-Higgs doublet model and light charged Higgs boson phenomenology. JHEP 1307, 044 (2013). arXiv:1212.6818 [hep-ph] 